\title{
Flagging and Correction of Pattern Noise in the Kepler Focal Plane Array.
}

\author{
Jeffery J. Kolodziejczak*a, Douglas A. Caldwell ${ }^{\mathrm{b}}$, Jeffrey E. Van Cleve ${ }^{\mathrm{b}}$, Bruce D. Clarke, \\ Jon M. Jenkins, ${ }^{b}$ Miles T. Cote ${ }^{b}$, Todd C. Klaus ${ }^{b}$, Vic S. Argabright ${ }^{\mathrm{c}}$ \\ ${ }^{a}$ Space Science Office, VP62, NASA Marshall Space Flight Center, Huntsville AL USA 35812; \\ ${ }^{\mathrm{b}}$ SETI Institute/NASA Ames Research Center, MS 244-30, Moffett Field, CA USA 94035; \\ ${ }^{\mathrm{c} B a l l}$ Aerospace \& Technologies Corp., 1600 Commerce Street, Boulder, CO USA 80301
}

\begin{abstract}
In order for Kepler to achieve its required $<20$ PPM photometric precision for magnitude 12 and brighter stars, instrument-induced variations in the CCD readout bias pattern (our "2D black image"), which are either fixed or slowly varying in time, must be identified and the corresponding pixels either corrected or removed from further data processing. The two principle sources of these readout bias variations are crosstalk between the 84 science CCDs and the 4 fine guidance sensor (FGS) CCDs and a high frequency amplifier oscillation on $<40 \%$ of the CCD readout channels. The crosstalk produces a synchronous pattern in the $2 \mathrm{D}$ black image with time-variation observed in $<10 \%$ of individual pixel bias histories. We will describe a method of removing the crosstalk signal using continuously-collected data from masked and over-clocked image regions (our "collateral data"), and occasionally-collected full-frame images and reverse-clocked readout signals. We use this same set to detect regions affected by the oscillating amplifiers. The oscillations manifest as time-varying moiré pattern and rolling bands in the affected channels. Because this effect reduces the performance in only a small fraction of the array at any given time, we have developed an approach for flagging suspect data. The flags will provide the necessary means to resolve any potential ambiguity between instrument-induced variations and real photometric variations in a target time series. We will also evaluate the effectiveness of these techniques using flight data from background and selected target pixels.
\end{abstract}

Keywords: Kepler, photometer, CCD, noise, pattern noise, CCD readout, crosstalk, image analysis, photometry

\section{INTRODUCTION}

Launched on March 6, 2009, the $31 / 2$ year Kepler Mission ${ }^{1}$ is to detect transiting exoplanets and determine the frequency of Earth-size planets in the habitable zones of sun-like stars. Previously, authors have described mission design and overall performance ${ }^{2}$, photometer design ${ }^{3,4}$, in-flight instrument performance ${ }^{5,8}$, and the overall data processing scheme ${ }^{6}$. Caldwell et al. ${ }^{4}$ describes several nonstationary image artifacts that are present in Kepler data and discusses their impact on photometric precision. This paper describes a method for recognizing and removing or flagging some of these artifacts. Specifically, the content includes a derivation of the need to develop this capability, a description of the methods employed and a summary of the results from applying it to a short period of flight data. Prior to this we provide a brief introduction to the instrument and data modes.

The Kepler focal plane consists of 84 separate science readout channels (identified as module\#.output\#) and four fine guidance sensor (FGS) channels all of which are read out synchronously as shown in Figure 1a. Each channel has several regions available to collect calibration, or "collateral" data (Figure 1b). There are two sets of columns of virtual pixels: (1) 12 columns of bias-only pixels resulting from 12 leading pixels in the serial register ("leading black"), and (2) a 20 column serial over-scan region ("trailing black"). There are also two sets of rows of collateral pixels: (1) the first 20 rows, which are covered by an aluminum mask ("masked smear"), and (2) a 26 row parallel over-scan region ("virtual smear"). During science data collection, a coadded sum of specified columns of the trailing black and rows of both the masked and virtual smear are stored at each cadence for each channel.

Science data are available at either short cadence ( $\sim 1$ minute) for 512 targets, or long cadence ( $\sim 30$ minutes $)$ for 170,000 targets. All science data are collected with an integration time of $6.02 \mathrm{~s}$ with pixels read out at a $3 \mathrm{MHz}$ clock rate. In science collection mode, the full single integration CCD frames are coadded together, then at the end of the short and long cadence period pre-specified pixels for each target are selected from the coadd, processed, and stored on board. Due 
to data storage and transmission limitations, only about $6 \%$ of the 96 million pixels are stored for eventual transmission to the ground.

Kepler's shutterless operation precludes standard dark frames. Instead, reverse-clocking of the CCDs permits us to measure the bias level throughout the image in the absence of sky signal. Also, a full frame image (FFI) mode permits collection of all the pixels in the focal plane. FFIs are used to examine detector properties, verify pointing, and verify the target aperture definitions. Reverse-clocked data and FFIs are taken periodically throughout the mission. ${ }^{\text {? }}$

a)

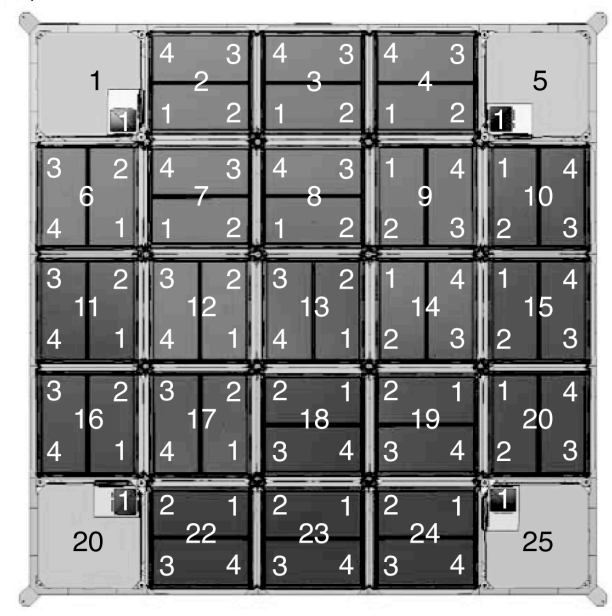

b)

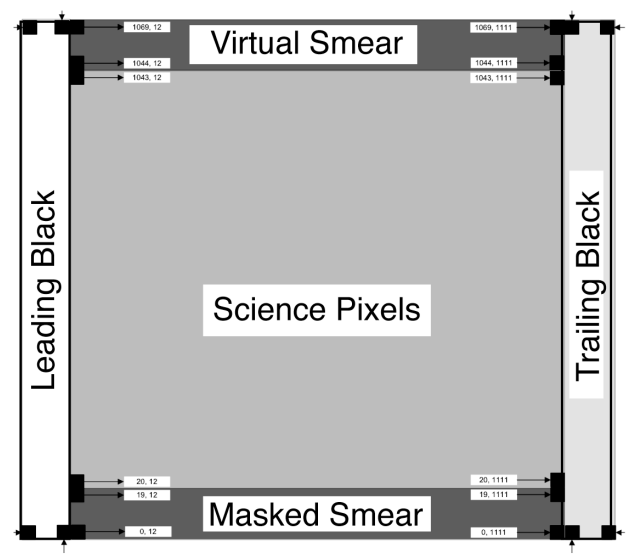

c)

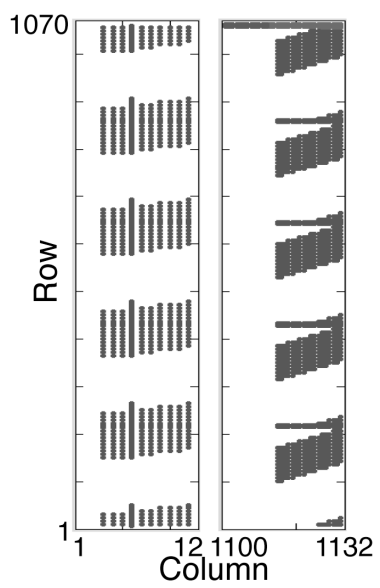

Figure 1. a) Focal plane with designations for modules 1-25, and outputs 1-4 for each module. Module.outputs 1.1, 5.1, 20.1 and 25.1 are fine guidance sensors (FGS). b) Identification of collateral regions associated with each output channel. c) Plots indicating the locations of artifact removal pixels (ARPs) in the leading and trailing black regions. These pixels are collected during each long cadence.

\section{OBJECTIVES}

To illuminate the motivation for this activity we begin with a brief description of the artifacts of concern. We identify the Kepler noise floor and relate this to the pattern noise levels or bias variability induced by the artifacts, defining an acceptable level for the bias variations. Our flagging and removal objectives are based on these levels.

\subsection{Description of artifacts}

Ground testing uncovered several instrumental artifacts, each of which was investigated to understand the cause, impact, and cost to fix or mitigate. These artifacts were extensively characterized on the ground and then again during commissioning. These investigations determined that several did not require mitigation. The existing data processing pipeline $^{6}$, or the algorithms described herein handle those with the largest impact. Those relevant to this paper are briefly described below and illustrated in Figure 2.

FGS Clocking Cross Talk. Cross talk from the FGS clocks to the science CCD video signals injects a complex pattern

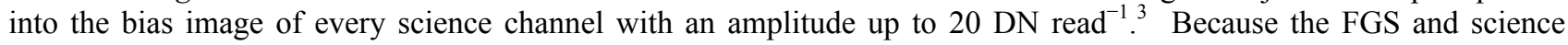
CCDs share the same master clock, the pattern is spatially fixed; however, the amplitude of the cross talk is dependent on the temperature of the Local Detector Electronics (LDE). The cross talk has three distinct components based on the state of the FGS CCDs as the science pixel is read out (see Figure 2a): FGS CCD frame transfer, parallel transfer, and serial transfer. Approximately $20 \%$ of targets have at least one of the parallel or frame-transfer cross talk pixels in their aperture. Without mitigation, the cross talk introduces a small time-varying bias into a target's flux time series as the LDE temperature changes.

High-frequency Oscillations. A temperature-sensitive amplifier oscillation at $>1 \mathrm{GHz}$ was detected in some CCD video channels during the artifact investigation. Investigation suggests that this signal may originate is from the AD8021 operational amplifiers used extensively in the video signal chain, which may show subtle layout-dependent instability when used at low gains. The oscillation's frequency range, rate of change, and pattern among the channels matched 
closely those characteristics in the dark images, strongly suggesting that the artifact is a moiré pattern generated by sampling the high-frequency oscillation at the $3 \mathrm{MHz}$ serial pixel clocking rate. Since the characteristic source frequency drifts with time and temperature of the electronic components by as much as $500 \mathrm{kHz} /{ }^{\circ} \mathrm{C}$, the signal from a given pixel in a series of dark images has a time varying signature. This signature may be highly correlated with neighboring pixels and yet poorly correlated with slightly more distant pixels. When the oscillation frequency is a harmonic of the serial clocking frequency, a DC shift occurs producing a horizontal band offset from the mean bias-level in the image. As the frequency drifts with temperature, the point on the image where this DC shift occurs moves up or down from sample-tosample, producing a rolling band.

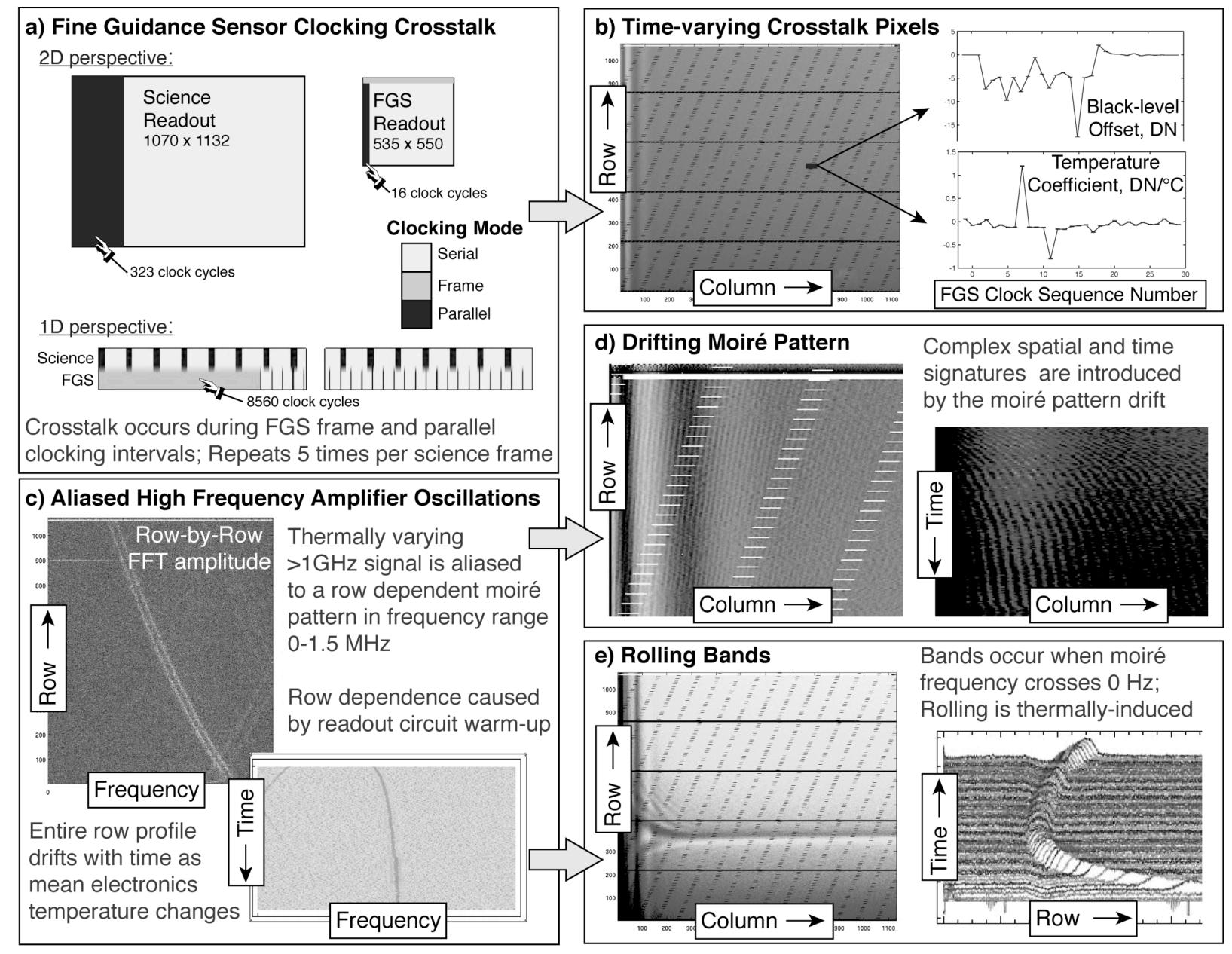

Figure 2. Illustrative description of pattern noise sources (left) and effects on Kepler images (right). a) The FGS pixels are read out synchronously with the science pixels but the difference in size of the two sensors combined with the changes in characteristics of the clocking signals during the parallel and frame transfer intervals produces b) a complex pattern of thermally varying pixels at specific locations across the image. c) An aliased high frequency signal is highly sensitive to LDE component temperature changes due to normal operational warm-up. These produce a smoothly varying frequency change with row. Note that a $0.1 \%$ change in the source frequency can produce an aliased frequency change from DC to Nyquist $(1.5 \mathrm{MHz})$ in the readout. One result is d) a moiré pattern with row-dependent frequency (left). The trace of a single row with time shows drift in the frequency (right). The other result is e) bands which occur when the aliased frequency crosses $0 \mathrm{~Hz}$, extend over all columns (left). The time-evolution of a single column shows rolling band in response to temperature changes (right). Plots are meant to show typical qualitative behavior for affected channels, so scales and data sources are suppressed. For reference b) shows flight data from output 20.2, c) shows ground test data from output 14.4, d) shows ground test data from output 9.3 and e) shows ground test data from 13.4.

Forty-six of the 84 readout channels have never exhibited moiré pattern behavior and an additional 9 channels have thus far not exhibited this behavior at a detectable level in flight. Ten channels have shown rolling bands. While the moiré 
amplitude per pixel in the remaining channels is significant, its affect on our ability to detect small planets depends on frequency, sum within a target aperture, and variations over time-scales of interest to transit detection. Based on the first 33.5 days of data from science operations ${ }^{9}$ (2010a) find the instrument is meeting the 6-hour precision requirement across the focal plane for the quietest $30 \%$ of stars. The two worst moiré channels, 9.2 and 17.2 , exhibit a $\sim 20 \%$ increase in 6-hour noise over the focal plane average at 12th magnitude, as measured by the standard deviation of 6-hour binned flux time series. Such an increase is small compared with the factor of 1.5 spread in the distribution of dwarf star precision at 12 th magnitude. ${ }^{9}$

For completeness, we briefly mention several other instrumental features in the Kepler data which are either accounted for in the current calibration scheme $e^{9}$ or are not currently observed to be variable enough to adversely affect Kepler science. The list includes: 1) Time-varying low-spatial-frequency characteristics, whereby the row-profile of the 2Dblack image tends to evolve slowly with time. 2) $L D E$ Undershoot, whereby star-like images induce a signal-dependent trailing undershoot in the video output, and 3) Start-of-line Ringing, whereby a transient signal is initiated at the onset of serial clocking of each row. the variations at low spatial frequency are accounted for in the current calibration using a cadence-by-cadence fit to the trailing black collateral data. A similar fit is used in the algorithms described in section 3 which also accounts for FGS crosstalk and LDE undershoot. This algorithm is designed to replace the current calibration method in a future pipeline release. An undershoot correction is also included in the current calibration pipeline with a model accounting extending up to 20 pixels when they are included in the target table. ${ }^{6}$ We have observed, and continue to expect the start-of-line ringing to be sufficiently stable over time to avoid photometric precision degradation.

Finally, scene dependent artifacts arise in two possible ways: 1) as a consequence of the sensitivity of the oscillating LDE component to temperature, the thermal transient introduced during readout by the signal from a bright star causes additional localized changes in the detected moiré pattern amplitude and frequency, and 2) time variability of pixels detecting bright stars, especially at the ends of saturated column segments, the LDE undershoot from beyond 20 pixels or from undetected pixels causes time variability in the black level. These scene dependent artifacts may persist over a range of hundreds of pixels in the columns following bright stars. In the 29 output channels exhibiting moiré pattern, approximately $20 \%$ of the FOV may be affected by these artifacts above the $0.02 \mathrm{DN} \mathrm{read}^{-1}$ pixel $^{-1}$ level. The undershoot effect impacts all channels at a much lower, but currently undetermined level. We will address these in future development if they are found to warrant high enough priority relative to other factors influencing photometric precision.

\subsection{Kepler's noise floor}

The Kepler design placed the noise floor for detecting stellar variation at a level that enables detection of earth sized planets in habitable orbits around 12th magnitude stars. The earth would produce an $84 \mathrm{ppm}$ decrement during a $12 \mathrm{hr}$ solar transit, and to ensure high detection efficiency for similar pairs of objects, a noise level of $20 \mathrm{ppm}$ for 6.5 hour periods was allocated. Artifact-induced changes in bias level which are above $20 \mathrm{ppm}$ on this time scale or longer have the potential to be confused with the signals resulting from stellar variability. The objective of this work is therefore to correct variations in biases to below the noise floor where possible and to detect and flag them where correction is not possible. Table 1 summarizes the implications in terms of the acquired signal levels in electrons and DN.

Table 1. Quantities used to define the level at which artifacts begin to affect Kepler science.

\begin{tabular}{|l|c|c|c|}
\hline Stellar magnitude (G-type star) & 11 & 12 & 13 \\
\hline $\begin{array}{l}\text { Detected in-aperture electrons in 6.5 hrs, aperture sizes are } \\
\text { typical for the magnitude }\end{array}$ & $\begin{array}{c}1.12 \mathrm{e}+10 \text { in } 25 \\
\text { pixel aperture }\end{array}$ & $\begin{array}{c}4.47 \mathrm{e}+09 \text { in } 16 \\
\text { pixel aperture }\end{array}$ & $\begin{array}{c}1.79 \mathrm{e}+09 \text { in } 9 \\
\text { pixel aperture }\end{array}$ \\
\hline Earth-equivalent decrement (electrons) & 939000 & 376000 & 150000 \\
\hline Allocated noise including shot noise (electrons) & 168000 & 89400 & 51900 \\
\hline $\begin{array}{l}\text { Bias change matching allocated noise level in aperture in 6.5 } \\
\text { hrs (electrons /pixel /read) }\end{array}$ & 1.9 & 1.6 & 1.7 \\
\hline Bias in DN, nominal 100 e/DN scale factor (DN/pixel/read) & 0.019 & 0.016 & 0.017 \\
\hline
\end{tabular}

The characteristics of pattern noise which justifies the additional attention are that it may be spatially correlated and temporally correlated, whereas ordinary white noise or other broadband types of noise are likely to be less so. For an aperture with 16 pixels, observed for a period of 6.5 hours (13 long cadences) noise will grow in the sum at a rate $\sqrt{16 \times 13} \approx 14$ times more slowly than a spatially and temporally correlated bias change. On the other hand, pattern 
characteristics vary widely, so only a small fraction of the time $\times$ field-of-view product is likely to be subjected to a reduction in sensitivity or increased likelihood of false detection. In this paper we use the term "source coverage" to identify the product of time $\times$ field-of-view.

\subsection{Acceptable bias variations}

It is clear that space and time correlated signals below $0.02 \mathrm{DN} /$ read over time intervals shorter than 6.5 hours would be difficult to distinguish from noise and therefore have little scientific impact. Typically, the acceptable level of bias variation would be constrained by detectability to several sigma above the noise level, however in this case we are able to leverage from the similar behavior of pixels acquired at a specific FGS clocking interval or pixels in an extended region of a given readout channel to measure bias variations at several times below the noise level. Since it is no more difficult to observe effects at the noise level than several times above, we simply round the $0.016 \mathrm{DN} \mathrm{read}^{-1} \mathrm{pixel}^{-1}$ to $0.02 \mathrm{DN} \mathrm{read}^{-1}$ pixel $^{-1}$ which is equivalent to $25 \mathrm{ppm}$ of the signal from a 12th magnitude star. Thresholds for correction and flagging of artifact-induced bias variations are based on limiting changes to $0.02 \mathrm{DN}$ read $^{-1}$ pixel $^{-1}$.

\subsection{Removal and flagging objectives}

FGS crosstalk is clearly detectable in data from collateral regions collected during every long cadence. The multiple examples of pixels collected during each FGS parallel and frame clocking interval typically indicate a repeating pattern which shows little or no change over a science readout. It is therefore possible to measure these bias changes on a cadence-by-cadence basis and have high confidence that the science pixels are subject to the same effects. The objective for the FGS algorithms is therefore to remove the FGS crosstalk signal to the level of $0.02 \mathrm{DN} \mathrm{read}{ }^{-1} \mathrm{pixel}^{-1}$.

Collateral data from smear regions also provides a way to measure the amplitude and frequency of moiré pattern on a cadence-by-cadence basis at the beginning and end of each readout interval. Relating this to the row-by-row amplitude and frequency of moire pattern in the difference between FFIs provides and indirect means of estimating the characteristics of moiré pattern at any readout location and time. Changes in trailing black collateral provide a similar means to detect and characterize rolling bands. For these however the confidence in the and exact estimate of the artifact-induced bias does not match that of FGS corrections. Such efforts are complicated significantly by complex phase variations and scene-induced changes in readout component temperatures, which lead to short-term changes in the oscillating frequencies and local shifts in the moire patterns. For these reasons, our objectives with respect to MPD and RBA are to flag regions of the focal plane and times when collateral data indicates that the amplitude of an effect is greater than $0.02 \mathrm{DN} \mathrm{read}^{-1}$ pixel $^{-1}$. The impact of moiré pattern with given amplitude on an aperture of given width in pixels, $n$, depends on the moire spatial frequency, $f$, on the peak-to-peak variation in an aperture is reduced by a factor $|2 \sin \pi f n / \pi f n|$. This makes it difficult to generalize the severity of the moire pattern in a flagged region independent of the target aperttures. Our approach is to flag data based on amplitude and provide localized severity information, such as frequency, which permits more precise evaluation of the impact on a given target.

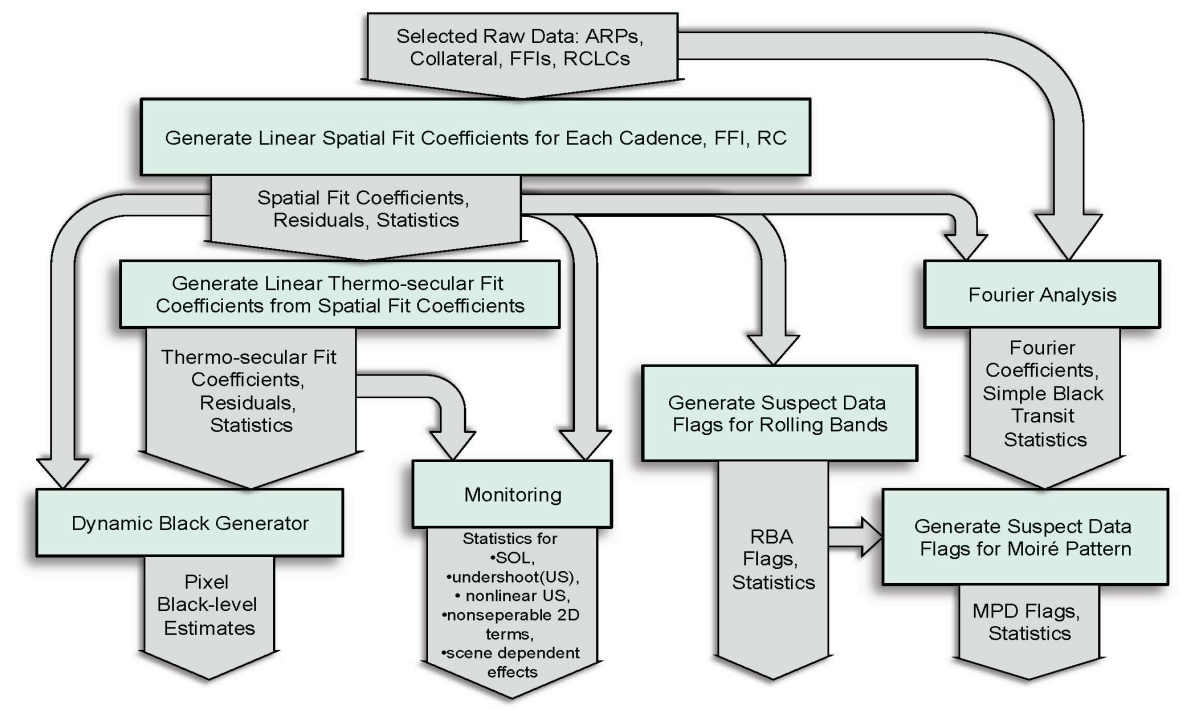

Figure 3. Architecture diagram showing prototype elements as boxes and data product flow as arrows. 


\section{METHODS}

The process developed to mitigate the effects of the pattern noise sources on Kepler science includes the elements and data products shown in figure 3.

\subsection{Spatial fitting}

The spatial fitting algorithm is designed to extract information about the time-varying parts of the Kepler data stream using pixel and collateral data from each cadence or FFI. We derive the information in the form of fit coefficients and uncertainties based on a model of the observed behavior of each pixel. Let $X_{R C}$ represent the raw black level value in $\mathrm{DN}$ at row $R$ and column $C$, and let $\mathrm{Z}$ represent the zero offset introduced to prevent negative values. Then we assume,

$X_{R C}-Z=X_{0}+f_{R C}+g_{C}+W_{R C}^{F}+W_{R C}^{P}+U_{R C}$

where,

$X_{0}$ is a constant

$f_{R C}$ is the row dependent part, (C only distinguishes leading and trailing black)

$g_{C}$ is the column dependent part

$W_{R C}^{F}$ is the FGS frame crosstalk dependent part

$W_{R C}^{P}$ is the FGS parallel crosstalk dependent part

$U_{R C}$ is the undershoot dependent part

Each of these components is defined in the Appendix in terms of linear coefficients. The fit values of all the coefficients and associated statistics are the data products of this algorithm.

The process includes initialization and fitting segments. Initialization of FGS clock states as a function of pixel location, selection of scene dependent exclusion zones, initialization of model components are parts of the initialization segment. Fitting the average of specified cadences and fitting each of a series of single cadences comprise the fitting segment.

The FGS frame and parallel crosstalk components occur in repeating patterns defined by the parallel and frame clocking intervals of the of the FGS. The term "row" applies to one of 1070 rows of a science channel image, each 1132 pixels long. The actual number of $3 \mathrm{MHz}$ clock cycles in a row is 1455 , so 323 clock cycles don't result in pixels (science parallel clocking). There are 5- 311370 clock-cycle FGS frames for every science frame, so an overall pattern repeats every 214 rows. In terms of the continuous clock stream, the frame crosstalk signal repeats every 16 pixels for 8450 clock cycles. It then flat-lines to a constant offset for 109 clock cycles followed by a 3-clock cycle transient signal. The parallel crosstalk signal repeats every 566 clock cycles with the first 16 cycles having the strongest crosstalk signal followed by a another 12-24 cycles which exhibit low-level transient variations the remainder of the 566 may be treated as constant except the last one which shows variability in some channels (cycles 17-566 are actually the serial clocking intervals of the FGS). The parallel pattern begins at FGS clock cycle 8563 with parallel cycle number 3 of 566 and continues mod 566 through the entire FGS clock cycle range to 311370.

To serve the fitting algorithm we assign the FGS-frame clock sequence numbers 1-16 to the repeating frame pattern, the value 17 to the 109 following pixels and 18-20 to the cycle ending transient all the remaining. The remainder of the 311370 are assigned the value 0. Similarly, we assign the FGS-parallel clock sequence numbers 1-566 to the repeating parallel pattern starting with value 3 assigned to the 8563 rd cycle and all preceding cycles assigned the value 0 .

After stacking the 5 FGS frames and chopping off the excess 323 unobserved columns, this provides a pair of mapping from (row, column) to FGS-frame clock sequence number and (row, column) to FGS-parallel clock sequence number. We fit a spatial model in which each pixel's black level is a separable function of row, column, FGS-frame clock sequence number (frame-CSN), and FGS-parallel clock sequence number (parallel-CSN). The algorithm allows fitting any combiations of frame and parallel values but we have limited the parallel FGS modeling range to pixels 1-29 and 565-66. The range 30-564 does not appear to contain time varying features.

Regions are excluded from the fits because they are potentially affected by moiré pattern drift changes or undershoot produced by signals from bright stars near the trailing black. These are apparently the result of temperature change induced by a the signals from bright stars on the readout circuit. For brightest stars, the signal may smear over many rows and therefore the scene dependent region extends over many rows. The algorithm used for selection of scene dependent exclusion zones is based on FFI images, based on a choice of signal threshold above which a pixel is 
considered potentially scene-dependent artifact inducing in DN/read, column threshold above which a pixel is considered potentially scene-dependent artifact inducing in the trailing black if it exceeds the signal threshold and the row pad count to extend each contiguous region to account for potential variations in star signal levels. Essentially, if a row of a robust averaged FFI contains a pixel above the signal threshold at a column beyond the column threshold, then any row within the pad count of that row will be excluded from the trailing black fits.

Only specific regions of an image are suitable for determining spatial coefficients. To systematize the selection of these regions the algorithm accepts specifications of a series of rectangular regions of interest (ROI) which can be thought of as building blocks for the complete modeled image region, containing pixel or collateral point attributes needed to assemble the modeled response vectors and predictor matrices. These summarized in Table 2.

Table 2. Regions of interest containing data used for black level spatial fitting. The 'type' column simply identifies the source of response vector data, "ARP" means artifact removal pixels from target LC data, "Collateral" means summed collateral data, "FFI" means FFI pixel data.

\begin{tabular}{|l|c|c|c|c|c|}
\hline ROI & min row & max row & min col. & max col. & type \\
\hline Leading ARP & 7 & 1059 & 3 & 12 & ARP \\
\hline Trailing ARP & 7 & 1051 & 1115 & 1132 & ARP \\
\hline Trailing ARP Undershoot & 1052 & 1063 & 1113 & 1132 & ARP \\
\hline Trailing Black Collateral & 7 & 1059 & 1119 & 1132 & Collateral \\
\hline Trailing FFI & 7 & 1063 & 1113 & 1132 & FFI \\
\hline
\end{tabular}

The complete model is assembled from a set of components. To systematize the construction of these components we identify represent distinct namable terms in the model which can be thought of as building blocks for the complete modeled predictor matrix, and associated indices for exclusion of potentially scene-dependent rows as a function of channel and all-zero columns in the design matrix. These are essentially the components of $X_{R C}$ described at the beginning of this section. There are also "delta" components measure the difference between leading and trailing black as detailed in the appendix. The design matrix is constructed with one row of information for each element of acquired data within each ROI model described in the appendix.

Prior to performing the cadence-by-cadence fitting for a given channel, we fit the pixel-by-pixel average of all specified cadences to obtain the exponential time constant parameter for the row-dependent exponential term using a nonlinear model and then perform a linear fit on the mean of pixel values over selected LCs to produce a set of mean coefficients. The single exponential time constant is used for all single-cadence fits for a given channel.

Finally, we perform cadence-by-cadence to obtain linear fit coefficients for individual cadences. The algorithm consists of the following steps:

- For each channel and for each long cadence:

- Select response vector data from input raw long cadences using the ROI indices described above.

- Determine cadence-specific undershoot component of design matrix which we apply to only a small subset of the modeled pixels as described in the appendix.

- Concatenate constant part of design matrix with undershoot component.

- Perform linear fit of scene-dependent-free data to obtain linear coefficients.

- Store linear fit results in output structures

- For each channel and for each FFI:

- Select response vector data from input raw FFI using the ROI indices described above.

- Determine FFI-specific undershoot component of the design matrix, includes all modeled pixels.

- Concatenate constant part of design matrix with undershoot component.

- Perform linear fit of all data to obtain linear coefficients.

- Store linear fit results in output structures 


\subsection{Thermo-temporal fitting}

The spatial fit for each cadence produces a time-series of spatial coefficients. These coefficients exhibit a variety of behaviors which are sometimes but not always simple functions of time or temperature. The purpose of the thermotemporal fitting algorithm is to fit the time series of each spatial coefficient, $C$, to the equation:

$C(t, T)=K_{0}+K_{t} t+K_{T} T$

where,

$K_{0}$ is a constant

$K_{t}$ is the linear trend in $C$

$K_{T}$ is the temperature coefficient of $C$

$t$ is time

$T$ is temperature.

In addition the algorithm provides the necessary statistics to evaluate whether the model fit is consistent with any of the possibilities $K_{t}=0, K_{T}=0$ or both $K_{t}$ and $K_{T}=0$. This evaluation is based on the $\chi^{2}$ of the four fit combination with zero or nonzero $K_{t}$ or $K_{T}$.

\subsection{D-black correction}

Not all the information from the spatial and thermo-temporal fits is typically required to adequately correct the data for the observed artifact-induced variations. Some of the fit parameters are intended only for monitoring instrument performance. The 2D black correction only includes the following terms.

$X_{R C}-Z=X_{0}+f_{R}+g_{C}+W_{R C}^{F}+W_{R C}^{P}$

where,

$X_{0}$ is a constant

$f_{R}$ is the row dependent part

$g_{C}$ is the column dependent part

$W_{R C}^{F}$ is the FGS frame crosstalk dependent part

$W_{R C}^{P}$ is the FGS parallel crosstalk dependent part

The undershoot and the "delta" components are treated as static, and monitored. In the event that time variability in these terms becomes significant enough in the future to impact science results, these terms could be added to the correction algorithm. The equation for $X_{R C}$ is separable in terms of the row, column and crosstalk dependent parts which allows the 2D corrections to be calculated from only the four vector terms, significantly shorting the processing time in comparison to an inseparable function.

The algorithm is implemented as a function with arguments a set of pixel rows, a set of pixel columns, a set of long cadence numbers which specify time and a parameter which determines whether the row and column lists should be interpreted as defining a discrete set of points or a rectangular region. The model is assembled from the spatial and thermo-temporal fit coefficients based on a decision tree. If the $\chi^{2}$ of the thermo-temporal fit indicates that the time series of the given spatial coefficient is not excluded from being a specific model at the $95 \%$ confidence level, then the spatial coefficient is determined from that model for all specified LC. In the event that multiple models meet the criteria, the preference order for the models, from highest to lowest is: constant, constant+linear-thermal, constant+linear-trendwith-time, constant+linear thermal +linear-trend-with-time. If none of the cases meet this criteria, a comparison between the standard deviation of the differences between consecutive coefficients in the time series and the formal errors in made. If the standard deviation of the differences is more than 1.5 times larger than the standard errors in the individual coefficients, as determined in the least-squares spatial fits, then the coefficients are applied discretely, otherwise they are smoothed using a quadratic thermo-temporal fit over an adaptive interval.

\subsection{Flagging}

The RBA and MPD share the same source signal, the high frequency amplifier oscillation described above, however RBA are somewhat simpler to identify. The effects of RBA on pixel time series are generally be larger and may be a 
greater risk to complicate the search for planet signatures. For these reasons, we have developed separate algorithms for detection and flagging of each.

Initially, overall datasets are divided into subsets defined by a range of rows, a range of columns, and a range of long cadences. Both algorithms share the same general flow involving the following steps:

Measurement: A measurement algorithm operates on each data subset to determine parameters indicating the presence of unusual bias level behavior. The accumulation of these parameters over the entire data set provides input to detection algorithms.

Detection: Detection algorithms compare the measured parameters with acceptability thresholds to detect unacceptable artifact behavior. The result is a map determining which parts of the full data set exceed the threshold criteria.

Postprocessing: Postprocessing algorithms accomplish two main tasks, a filter to clean spuriously flagged regions and a padding algorithm to add a buffer zone around areas a high density of above threshold flags. The former prevents unnecessary flagging of data which is not at significant risk to interfere with planet search algorithms. The later accounts for the fact that we do not directly measure the science pixels but rather collateral data which is indicative of the science pixels, and thus there is some level of uncertainty in boundaries of affected locations. A final step in the postprocessing identifies rectangular boundaries in the padded flagged regions to be listed as suspect data flags objects to be carried forward as input to pipeline algorithms or as data products supplied to users.

Severity evaluation: A final algorithm assembles a set of statistics which characterize the severity of the flagged artifacts in the identified regions. The algorithms operate on the data corresponding to the boundaries derived for the suspect data flag objects to produce a set of parameters describing the pixels and cadences being flagged by that object. The flags are paired with the severity parameters to form a list which represents the primary output of the flagging algorithms. The distinctions between the two algorithms are summarized in the Table 3 with further detail in the ensuing paragraphs.

Table 3. Flagging algorithm implementation comparison between rolling band flagging and moiré pattern drift flagging.

\begin{tabular}{|c|c|c|}
\hline Item & RBA & MPD \\
\hline Measurement & $\begin{array}{l}\text { - time-varying displacement in trailing } \\
\text { black spatial fit residuals, not due to } \\
\text { scene dependent artifacts } \\
\text { - black transit search in trailing black } \\
\text { residuals and smear differences }\end{array}$ & $\begin{array}{l}\text { - Amplitude of moiré pattern in } 19110- \\
\text { column subregions } \\
\text { - frequency of moiré pattern } \\
\text { - black transit search in trailing black } \\
\text { residuals and smear differences }\end{array}$ \\
\hline Detection & $\begin{array}{l}\text { - displacement sigma vs. time }>0.02 \\
\text { DN/pixel/read } \\
\text {-or - } \\
\text { - both fixed residual displacement and } \\
\text { black transit }>0.02 \mathrm{DN} / \text { pixel/read }\end{array}$ & $\begin{array}{l}\text { - moiré pattern amplitude }>0.02 \\
\text { DN/pixel/read in subregions } \\
\text {-or- } \\
\text { - black transit }>0.02 \mathrm{DN} / \text { pixel/read }\end{array}$ \\
\hline Postprocessing & $\begin{array}{l}\text { - filter out flag densities less than } 5 \% \text { in } \\
10 \text { row by } 39 \text { LC region } \\
\text { - pad around surviving flags } \pm 19 \text { LC } \\
\text { and } \pm 10 \text { rows } \\
\text { - package flagged regions into } \\
\text { rectangular suspect data flags (SDF). }\end{array}$ & $\begin{array}{l}\text { - filter out flag densities less than } 5 \% \text { in } 10 \\
\text { row by } 39 \text { LC region } \\
\text { - pad around surviving flags } \pm 18 \text { LC and } \\
\pm 10 \text { rows } \\
\text { - package flagged regions into rectangular } \\
\text { suspect data flags (SDF). }\end{array}$ \\
\hline Severity Evaluation & $\begin{array}{l}\text { measure statistics of measured } \\
\text { parameters within each SDF region. } \\
\text { - } 97.7 \text { percentile \& median displacement } \\
\text { and fraction of exposure exceeding }\end{array}$ & $\begin{array}{l}\text { measure statistics of measured parameters } \\
\text { within each SDF region. } \\
\text { - } 97.7 \text { percentile \& median peak-to-peak } \\
\text { moiré spatial amplitude and fraction of }\end{array}$ \\
\hline
\end{tabular}




\begin{tabular}{|c|c|c|}
\hline & $\begin{array}{l}\text { displacement threshold } \\
\text { - } 97.7 \text { percentile \& median noise and } \\
\text { fraction of exposure exceeding noise } \\
\text { threshold } \\
\text { - } 97.7 \text { percentile \& median bias variation } \\
\text { and fraction of exposure exceeding bias } \\
\text { variation threshold } \\
\text { - number of }>3 \&>4 \text { sigma transit-like } \\
\text { features } \\
\text { - total fraction of exposure exceeding } \\
\text { combined thresholds }\end{array}$ & $\begin{array}{l}\text { exposure exceeding amplitude threshold } \\
\text { - median spatial frequency at peak } \\
\text { amplitude. } \\
\text { - } 97.7 \text { percentile \& median bias variation } \\
\text { and fraction of exposure exceeding bias } \\
\text { variation threshold } \\
\text { - number of }>3 \&>4 \text { sigma transit-like } \\
\text { features } \\
\text { - total fraction of exposure exceeding } \\
\text { combined thresholds }\end{array}$ \\
\hline
\end{tabular}

$R B A$. The signature of a rolling band is a time-varying displacement in trailing black spatial fit residuals, not due to scene dependent artifacts. The algorithm searches for these on a row-by row basis for each channel. It also convolves a square wave transit kernel with these time series as well as the column-by-column difference between masked and virtual smear time search for places where the black level variations exhibit transit-like signature. We call these black transits. Clearly if the collateral data is introducing transit-like time signatures into the data, we want to make sure it gets flagged even if the typical variation in the pixel is small.

$M P D$. The MPD flags require additional processing steps which include fast Fourier transforms of both the difference between a pair of FFIs and the difference between masked and virtual smear collateral data for each long cadence. The moiré pattern produces a detectable trace of high amplitudes with a continuous frequency signature as shown in figure 6 . The FFI gives a spatial distribution of moiré amplitudes while the smear differences give variation in time. By matching the smear frequencies in the FFIs to the nearest long cadence and then scaling the spatial distribution on a cadence-bycadence basis we can estimate the moiré pattern amplitude in any row at any time. FFTs are also taken in 19110 column-wide subregions at 54 column steps to measure the column dependence of the amplitude as well.

\section{RESULTS}

We applied these algorithms to a cross-section of Kepler flight data. The following paragraphs describe the results of these prototype runs. Table 4 identifies the flight data used to exercise each algorithm. The channels 2.1 and 12.1 were selected as "good channels", 6.2 and 20.2 exhibit worst case FGS crosstalk, and 9.2 and 17.2 exhibit worst-case MPD.

Table 4. Module Outputs and time intervals used to exercise the various algorithms. Q0 lasted 10 days during commissioning from May 8-18, 2009, Q1 lasted 35 days from May 19-June 23, 2009.

\begin{tabular}{|l|c|c|c|}
\hline \multicolumn{1}{|c|}{ Item } & All Long Cadences Q0-Q1 & All LC first 10 days of Q1 & $\begin{array}{c}\text { Decimated set: every 10th LC } \\
\text { for Q0; every 20th LC for Q2 }\end{array}$ \\
\hline Fitting & $2.1,6.2,9.2,12.1,17.2,20.2$ & All & All \\
\hline Correction & $2.1,6.2,9.2,12.1,17.2,20.2$ & None \\
\hline RBA Flagging & $2.1,6.2,9.2,12.1,17.2,20.2$ & All & None \\
\hline MPD Flagging & $2.1,6.2,9.2,12.1,17.2,20.2$ & All & None \\
\hline
\end{tabular}

\subsection{Example fits}

Kepler readout channels exhibit a wide variety of black-level morphologies. This section illustrates the model response fidelity using a few typical channels which span the morphology range. Figure 4 shows the raw trailing black collateral data for two channels, 2.2 and 16.3 along with the fit curves. Some row regions were excluded from the fits because of bright stars near the trailing black, which would have introduced unmodeled scene dependent artifacts that would bias the fit coefficients if not explicitly removed. 

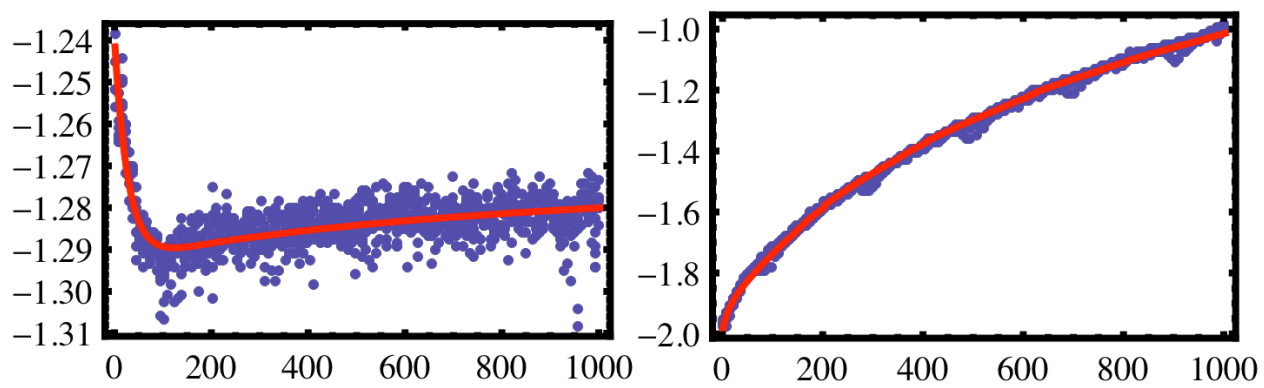

Figure 4. Example comparison of fit curves and data comparing low frequency vertical behavior of channels 2.2 (a) and 16.3 (b) for one representative Q0 long cadence. The scales are trailing black row vs. black-level in $\mathrm{DN} / \mathrm{pixel} / \mathrm{read}$, with arbitrary offset. The row dependence of the black level is modeled well by an exponential term with time constant which varies from channel to channel plus a logarithmic term which is channel independent.

The variation in the low frequency row-dependent terms of the model is evident from the two examples above. The series of spiked rows, repeating 5 times, are collateral rows containing FGS crosstalk sensitive pixels. The fit estimates offset values for each crosstalk-sensitive pixel from the combination of both the collateral row values representing the sum of 14 pixels and the individual ARPs. Undershoot coefficients represent filter coefficients for an additive term to each pixel based on a linear combination of the values of the previous 20. Figure 5 shows typical time dependent behavior of coefficients.

a)

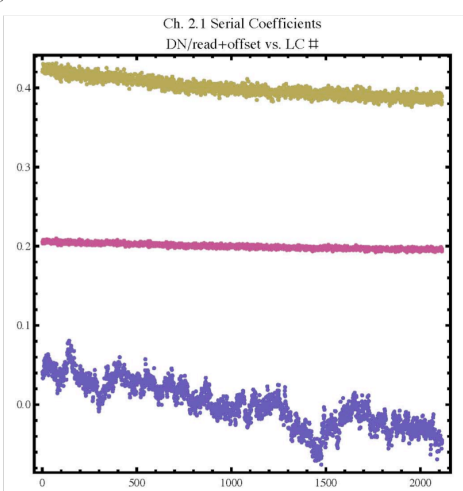

c)

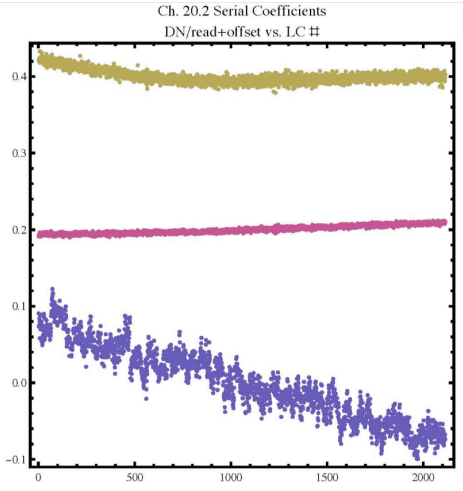

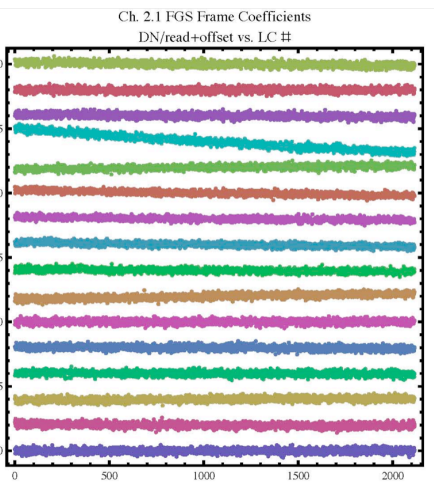

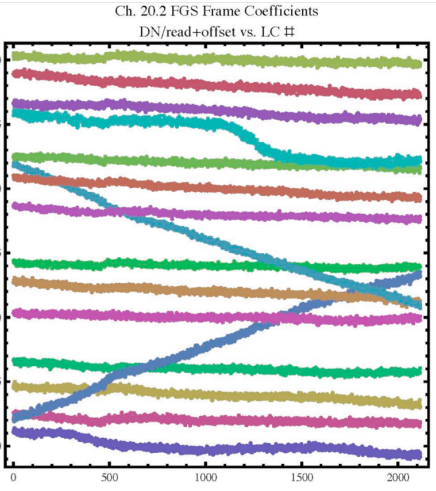

Figure 5. Examples of behavior of spatial fit coefficients vs. time for channels $2.1(a, b)$ and $20.2(c, d)$. a) and c) show the behavior of the low frequency spatial coefficients. The bottom traces show the constant term which is the only coefficient which always varies discretely (i.e. cannot be smoothed). The middle and top trace are the log and exponential coefficients respectively. b) and d) show the FGS frame crosstalk coefficients. The scales are $\mathrm{DN} / \mathrm{pixel} / \mathrm{read}$ vs. time as measured by long cadence number. An arbitrary offset has been added to each curve to provide visual separation. 


\subsection{Effect of corrections on targets}

Calibrated Kepler pixels are subject to 3 separate effects of FGS crosstalk stemming from a) the 1-D black correction, b) the smear correction and c) the effects of any FGS-crosstalking pixels in the target aperture. In the current crosstalkunaware 1-D black correction, a robust polynomial fit is applied to the trailing black collateral data. Even with a robust fitting algorithm there is likely to be some time varying bias introduced by crosstalking pixels with nonzero weights on the fit coefficients and thereby on the correction of each science pixel. $89 \%$ of collateral rows are unaffected by FGScrosstalking pixels. The introduced bias in a given row is applied to uniformly to all science pixels in that row. The crosstalk-unaware smear correction is a cadence-by-cadence column-dependent correction based on measurements from the masked and virtual smear collateral regions. Each smear region is the sum of 12 rows. $57 \%$ of collateral columns are unaffected by FGS-crosstalking pixels, and $\{22 \% \mid, 3 \%, 12 \%, 5 \%\}$ have $\{4,3,2,1\}$ modeled pixels in a given column. The introduced bias in a given column is applied to uniformly to all science pixels in that column. Both effects are reduced by the averaging and filtering afforded by the robust fits for the 1D black correction and the averaging over typically 24 rows for the smear correction.

The FGS-crosstalking pixels in the science pixel region represent the primary motivation for developing a dynamic 2D black correction. Target apertures currently have no time-varying correction for crosstalking pixels. $82 \%$ of targets contain no FGS-crosstalking pixels. In this case the introduced bias in a given pixel applies to only science pixels in that target aperture. The overall impact on the affected $18 \%$ of targets is substantially reduced by averaging over the whole aperture and the fact that the crosstalk is somewhat smaller and changes sign for some pixels thereby producing further dilution by averaging.

Figure 6 shows a histogram of the peak-to-peak dynamic 2D black corrections per target for quarter 1 where mean LDE temperature varied by $1^{\circ} \mathrm{C}$ over the full 35 day interval. The figure shows both a worst case channel, which is highly susceptible to crossstalk, and the cumulative impact on all channels. The separated effects FGS-crosstalking in the collateral regions versus the science pixel regions is shown for only the worst case channels. $12 \%$ of targets are affected by more than $0.02 \mathrm{DN} / \mathrm{pixel} / \mathrm{read}$ in the worst case, while only $3.4 \%$ of all targets are affected at this threshold level. The potential exists for a larger fraction targets to be affected by $0.02 \mathrm{DN} /$ pixel/read or more for full quarters, but because the temperature varies significantly more is this period, these values are likely to be typical of a quarter. It is noteworthy that the worst case average temperature coefficient for a target is $\sim 0.6 \mathrm{DN} /$ pixel $/{ }^{\circ} \mathrm{C}$ implying that a $0.03^{\circ} \mathrm{C}$ change in $\mathrm{LDE}$ temperature with the appropriate time signature would be required to produce the signature of an earth-sized planet. Even then, the thermal excursion would need to repeat 3 times at regular intervals to produce a false positive. A more likely possibility is that the less predictably varying pixels, as shown in figure 5 , will reduce sensitivity to transits for limited periods in a small fraction of targets. Nevertheless, the increased risk of unanticipated consequences that these simple-to-remove artifacts could have on planet detection or other astrophysical applications for Kepler data warrants including this correction algorithm in the ongoing evaluation process for Kepler science pipeline enhancements.

a) worst case channel 20.2

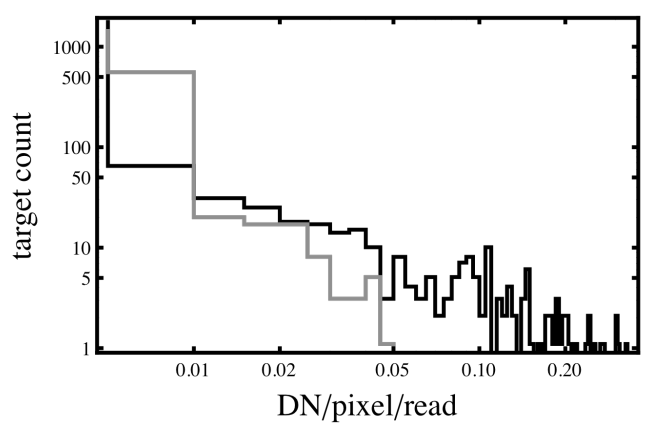

b) all channels

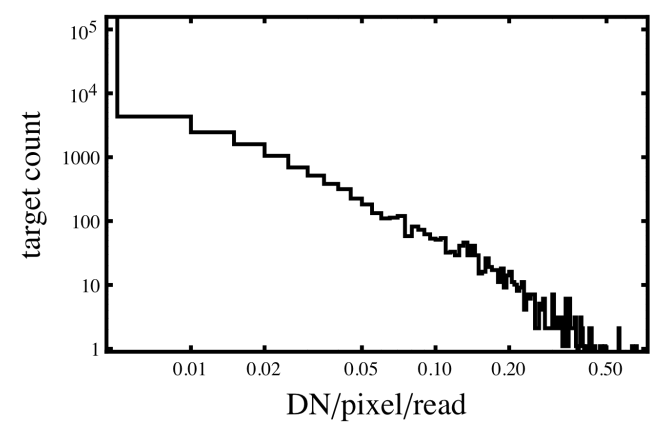

Figure 6. Histograms of peak-to-peak dynamic 2D black corrections per science target for a) worst case channel 20.2 and b) all channels during Q1. In (a) the contribution of collateral effects is shown in gray. 


\subsection{Flagging effectiveness}

Two examples of rolling bands detected and flagged by the algorithm described above are illustrated in figure 7 for the result of combining quarters 0 and 1 . The vertical axis is the row number, and the horizontal axis is time as measured by long cadence number. The varying shaded areas represent the degree beyond threshold level that the data indicates a rolling band, the white regions are the padding around the offending regions and the black areas are unflagged. The figure shows the movement of the rolling bands. The pattern in the signal over time at the top of figure 7.b is from a scene dependent region caused by a variable star near the trailing black region. When a scene dependent region is flanked by a rolling band, the flagging algorithm automatically flags the scene dependent region as part of the rolling band. If no rolling band is present, the flag remains scene dependent. The rolling band flags apply to all columns in a flagged row so we can define the rolling-band-free source coverage as the fraction of black cells in these 2D maps. Here we define "artifact-free source coverage" as the fraction of available field of view solid angle $\mathrm{x}$ exposure time that is unaffected by a given artifact. In the test cases below the rolling-band-free source coverage is $60 \%$ and $78 \%$ for module outputs 9.2 and 17.2 respectively. The rolling-band-free source coverage was $100 \%$ for all but 10 channels in the first 10 days of quarter-1 data.

a) Channel 9.2

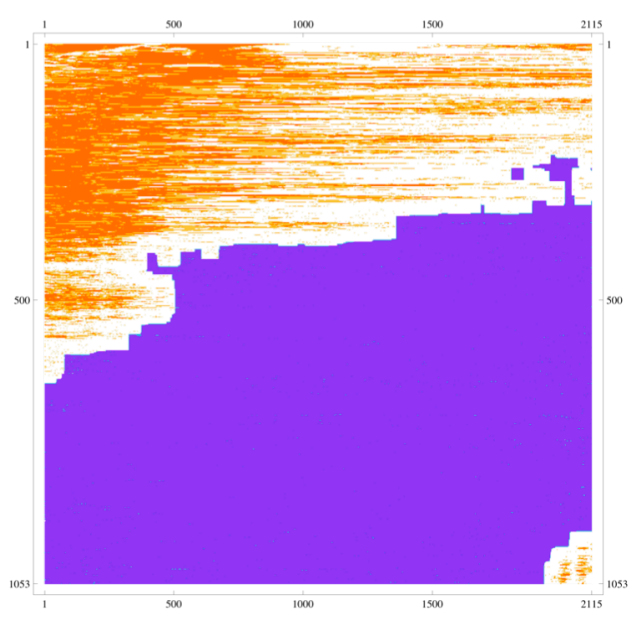

b) Channel 17.2

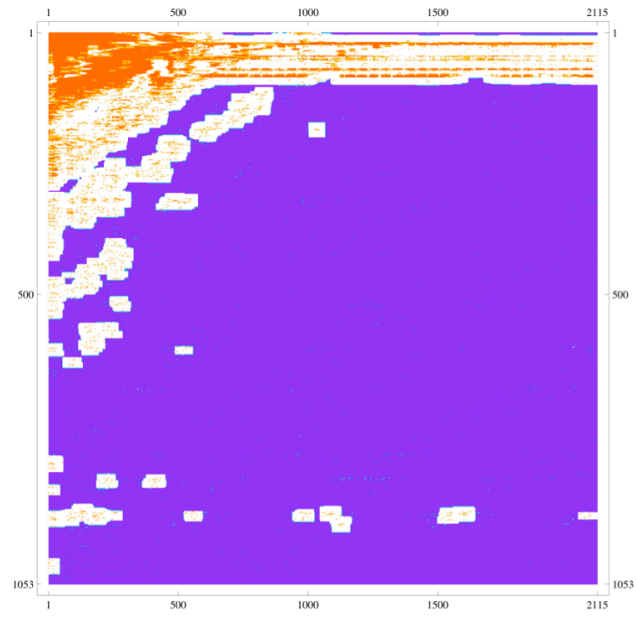

Figure 7. Example of rolling band flagging results from channels a) 9.2 and b) 17.2 for combined Q0-Q1. Varying shaded areas represent the degree beyond threshold level that the data indicates a rolling band, white regions are the padding around the offending regions and black areas are unflagged. The scales are image row vs. time as measured in long cadence periods.

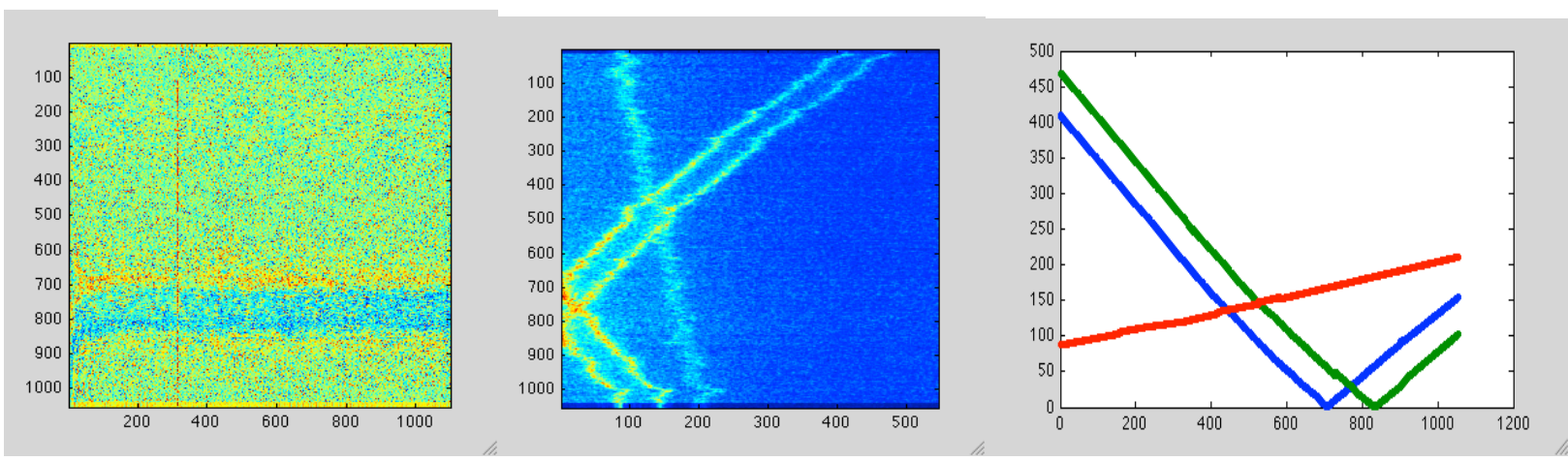

Figure 8. Example of moiré pattern detection algorithm. a) Difference between 2 full frame images from channel 17.2 during Q0. b) Row-by-row FFT of image difference indicating 3 frequency traces. The scales are FFT frequency bin vs. row. The FFT bins range from 0 to $1.5 \mathrm{MHz}$. c) The 3 frequency traces are detected by the filtering and detection algorithm which is designed to avoid confusion when traces cross. The plot is FFT frequency bin as a function of row, with FFT bins as in b). 
Effectiveness of the moiré pattern detection algorithm is illustrated in figure 8. The differenced FFI pair shown in figure 8.a shows clear moiré pattern features after subjection to a row-by-row FFT as shown in figure 8.b. The MPD detection and flagging algorithm identifies the distinct frequencies traces in the images (figure 8.c) and determines the amplitudes at each frequencies in each subwindow as discussed in the algorithm description. Similar algorithms generate traces for the difference between the masked and virtual smear for each long cadence to enable the frequency matching and amplitude scaling between full images and long cadences. These scaled amplitudes are used in the thresholding algorithm to flag data which is affected by moiré patterns.

When applied to all the readout channels, the algorithm detected peak-to-peak moiré amplitudes ranging from 0 to 1.2 $\mathrm{DN} /$ pixel/read over all possible moiré frequencies (1-1.5 MHz) . The free-pattern-drift-free source coverage was $100 \%$ for 65 module-outputs and $0 \%$ for 6 module-outputs during the first 10 days of quarter- 1 . The source coverage in the remaining 13 readout channels varied from $17 \%$ to $86 \%$.

\section{CONCLUSIONS AND FORWARD WORK}

The prototype algorithms described above successfully detect and correct or flag the pattern noise sources observed in the Kepler data stream. The following sections summarize the net impact of the image artifacts on the total Kepler field of view and outlines forward plans for algorithm implementation as well as mitigation of scene dependent artifacts.

\subsection{Summary}

a)

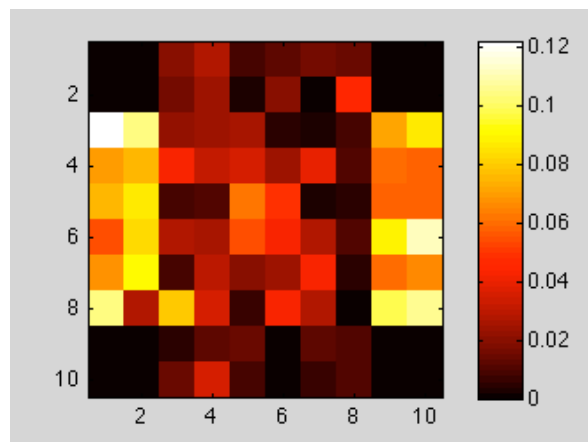

b)

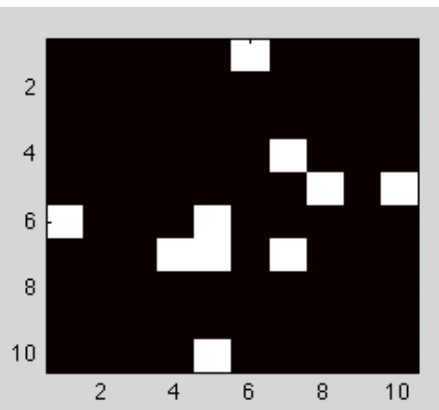

c)

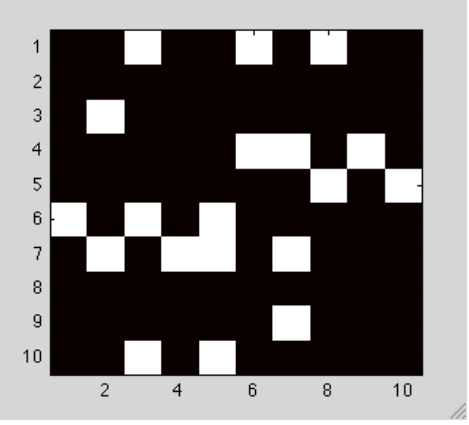

Figure 9. a) FGS cross-talk afflicted channels, with color indicating the fraction of targets require corrections of $0.02 \mathrm{DN} / \mathrm{pixel} / \mathrm{read}$ or greater. b) channels exhibiting rolling bands are highlighted in white. c) channels exhibiting moiré pattern drift are highlighted in white.

Results based on the first month of Kepler science data collection from all channels are consistent with prelaunch expectations. While $17.4 \%$ of targets' optimal apertures include modeled FGS clock intervals, only $3.7 \%$ of targets require corrections of $0.02 \mathrm{DN} / \mathrm{pixel} / \mathrm{read}$ or greater over the entire 35 day period during which the temperature changed by $1^{\circ} \mathrm{C}$. Rolling bands were evident on 9 channels during the first 10 days of this period, resulting in flagging of $3.0 \%$ of the field of view. The rolling bands move with time so the shorter interval gives a total flagged area representative of the affected size at any given instant. During the same interval moiré pattern afflicted 19 channels, resulting in $12.7 \%$ of the field of view being flagged. This value is $\sim 30 \%$ larger than prelaunch estimates and likely stems from the fact that temperatures are more stable and therefore the temperature profile during a single Kepler read is more repeatable. This probably leads to greater coherence in the co-added frames extending the moiré patterns further across the image rows. Figure 9 shows which Kepler channels are affected by the various pattern noise sources.

\subsection{Implementation in the Kepler Science Processing Pipeline}

Prior to launch artifact mitigation was considered a top priority for Kepler pipeline implementation. This plan is undergoing a reprioritization based on in-flight observations of photometric performance and evaluation of the effects of other systematic errors and corrections. As a result it is likely that these prototype algorithms will be delayed to a later 
pipeline development phase, assuming funding is available. Nevertheless, the completed prototype can de applied to any and all Kepler data to evaluate the impact of artifacts on planet detection and measured parameter uncertainties.

\subsection{Plans for scene dependent artifacts}

The next step in artifact mitigation is to evaluate the impact of scene dependent artifacts on the field of view. Certain artifact features are temporarily distorted by the thermal transients introduced into the sensitive readout circuits by bright stars which saturate pixels. These stars produce a smear pattern extending over several rows in a given column. Specifically, the moiré pattern frequency changes abruptly and the amplitude increases sharply for tens of pixels following these features. Pixels in the wake of these saturated segments are thereby subject to increased black-level variation. The affected regions are not directly detectable in the collateral regions and thus will require FFI images for detection. An algorithm which measures the Fourier peak amplitude in short spatial segments in these regions in differenced FFIs is expected to be effective at flagging the at-risk regions of MPD affected channels. In addition, variability of the bright stars changes the saturated segment's length producing a large dynamic range in the end pixels. Variation in these pixels produces variation in the downstream pixels in the same rows as a result of the undershoot. Since undershoot is only corrected in collected pixels, it would also be necessary to flag these areas based on FFI images and the known characteristics of bright variable stars in the field of view. In many cases it is possible to monitor the variability of the bright stars in the smear collateral regions on a continuous basis. Channels typically have only $\sim 500$ saturated and near saturated pixels so even if these effects extend for 100 pixels downstream in the readout chain of each bright pixel , $<5 \%$ of the field of view would be affected. Nevertheless the need for automation in the detection of planet candidates in the $>150000$ Kepler targets supports the need to flag these at-risk regions.

\section{APPENDIX}

This appendix defines the terms in the spatial model. We are modeling several discrete components with only the leading $(\mathrm{C} \leq 12)$ and trailing black so it will be convenient to define the following discrete delta functions:

$$
\begin{aligned}
& \delta_{X Y}=\left\{\begin{array}{ll}
1 & \text { if } X=\mathrm{Y} \\
0 & \text { if } X \neq \mathrm{Y}
\end{array}\right\} \\
& \delta_{C \in L B}=\left\{\begin{array}{ll}
1 & \text { if } \mathrm{C} \leq 12 \\
0 & \text { if } \mathrm{C}>12
\end{array}\right\} \\
& \delta_{\{R, C\} \in U S}=\left\{\begin{array}{ll}
1 & \text { if }\{\mathrm{R}, \mathrm{C}\} \in \text { undershoot ARPs } \\
0 & \text { otherwise }
\end{array}\right\} \\
& \delta_{\{R, C\} \in i}^{F}=\left\{\begin{array}{ll}
1 & \text { if }\{\mathrm{R}, \mathrm{C}\} \in \text { pixels with FGS frame sequence number } i \\
0 & \text { otherwise }
\end{array}\right\} \\
& \delta_{\{R, C\} \in i}^{P}=\left\{\begin{array}{ll}
1 & \text { if }\{\mathrm{R}, \mathrm{C}\} \in \text { pixels with FGS parallel sequence number } i \\
0 & \text { otherwise }
\end{array}\right\}
\end{aligned}
$$

With these we can define the various modeled terms for LC fits:

$$
\begin{aligned}
& f_{R C}=\left(C_{1}^{f}+\delta_{C \in L B} C_{2}^{f}\right) \ln \left(\frac{R}{R_{\mathrm{log}}}+1\right)+\left(C_{3}^{f}+\delta_{C \in L B} C_{4}^{f}\right) e^{-\frac{R}{R_{\mathrm{exp}}}} \text {, where } R_{\mathrm{log}} \text { and } R_{\mathrm{exp}} \text { are constants } \\
& g_{C}= \sum_{i \in C_{L B}} \delta_{C i} C_{i}^{g}+\delta_{C \in L B} C_{0}^{g} \text {, where } C_{L B} \text { is the list of discretely modeled leading black columns } \\
& W_{R C}^{F}=\sum_{i \in F G S-F} \delta_{\{R, C\} \in i}^{F}\left(C_{1, i_{F G S-F}}^{W F}+\delta_{C \in L B} C_{2, i_{F G S-F}}^{W F}\right) \text {, where FGS - F are modeled FGS frame clock states } \\
& W_{R C}^{P}=\sum_{i \in F G S-P} \delta_{\{R, C\} \in i}^{P}\left(C_{1, i_{F G S-P}}^{W P}+\delta_{C \in L B} C_{2, i_{F G S-P}}^{W P}\right), \text { where FGS - P are modeled FGS parallel clock states } \\
& U_{R C}=\delta_{\{R, C\} \in U S}\left(C_{0}^{U}+\sum_{j=C-N_{U S}}^{C-1} C_{C-j}^{U} X_{R j}\right), \text { where } X_{R j} \text { is a measured pixel signal value, and } N_{U S} \text { is the } \\
& \text { number of modeled undershoot columns. }
\end{aligned}
$$




\section{REFERENCES}

[1] Borucki, W. J., et al., "Kepler Planet-Detection Mission: Introduction and First Results," Science 327(5968), $977-$ (2010).

[2] Koch, D. G., et al., "Kepler Mission Design, Realized Photometric Performance, and Early Science ," ApJ. 713(2), L79-L86 (2010).

[3] Argabright, V. S., et al., "The Kepler photometer focal plane array 2008," Proc. SPIE, 7010, 76 (2008).

[4] Van Cleve, J., \& Caldwell, D. A., [Kepler Instrument Handbook], KSCI 19033-001 (Moffett Field, CA: NASA Ames Research Center), http://archive.stsci.edu/kepler/, (2009).

[5] Caldwell, D. A., et al., "Instrument Performance in Kepler's First Months," ApJ. 713(2), L92-L96 (2010).

[6] Jenkins, J. M., et al., "Overview of the Kepler Science Processing Pipeline," ApJ. 713(2), L87-L91 (2010).

[7] Haas, M. R., et al., "Kepler Science Operations," ApJ. 713(2), L115-L119 (2010).

[8] Caldwell, D. A., et al., "Kepler Instrument Performance: An In-flight Update," Proc. SPIE, 7731, (2010).

[9] Jenkins, J. M., et al., "Initial Characteristics of Kepler Long Cadence Data for Detecting Transiting Planets," ApJ. 713(2), L120-L125 (2010). 


\title{
Flagging and Correction of Pattern Noise in the Kepler Focal Plane Array
}

\author{
Presenter: Jeff Kolodziejczak ${ }^{a}$ \\ kolodz@nasa.gov \\ 256-961-7775
}

Co-Authors: Douglas A. Caldwell ${ }^{b}$, Jeffrey E. Van Cleve ${ }^{b}$, Bruce D. Clarke ${ }^{b}$, Jon M.

Jenkins $^{\mathrm{b}}$, Miles T. Cote ${ }^{\mathrm{b}}$, Todd C. Klaus ${ }^{\mathrm{b}}$, Vic S. Argabright ${ }^{\mathrm{c}}$

aSpace Science Office, VP62, NASA Marshall Space Flight Center, Huntsville AL USA 35812;

bSETI Institute/NASA Ames Research Center, MS 244-30, Moffett Field, CA USA 94035;

'Ball Aerospace \& Technologies Corp., 1600 Commerce Street, Boulder, CO USA 80301 


\section{Image Artifacts producing pattern noise}

- Artifacts are time-varying instrumental effects on science pixels

- Often readout electronics temperature dependent

mod.out: 20.2 virtual smear

Artifacts:

Deviation of Mean of Each Parallel FGS Crosstalk Pixel

- Thermally Varying FGS Crosstalk (TVFX)

- FGS frame transfer: Repeating pattern every 16 pixels

- FGS parallel transfer: patterns are nominally 16 pixels + an additional transient
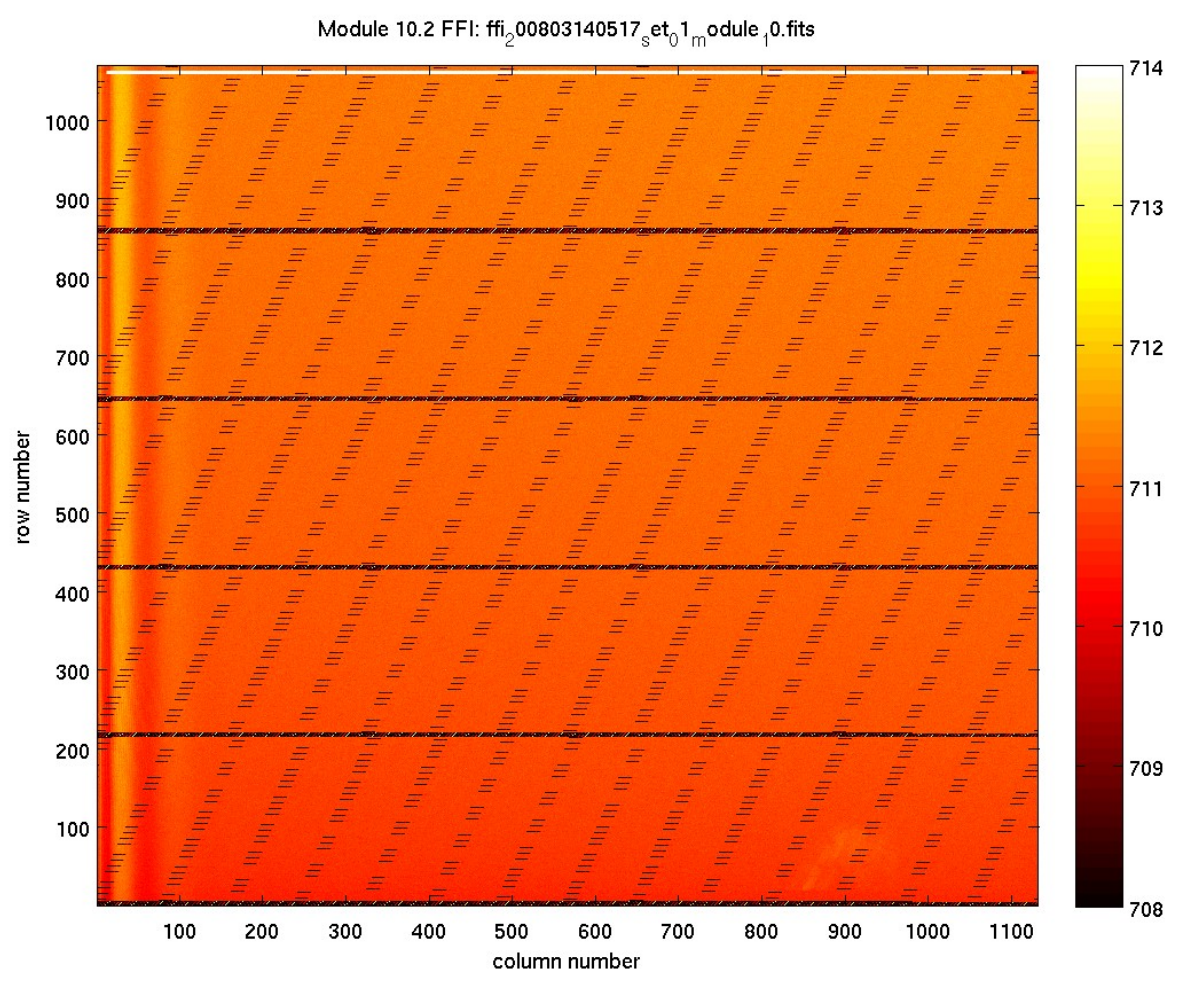
from Mean of 16 Pre-FGS Leading Pixels

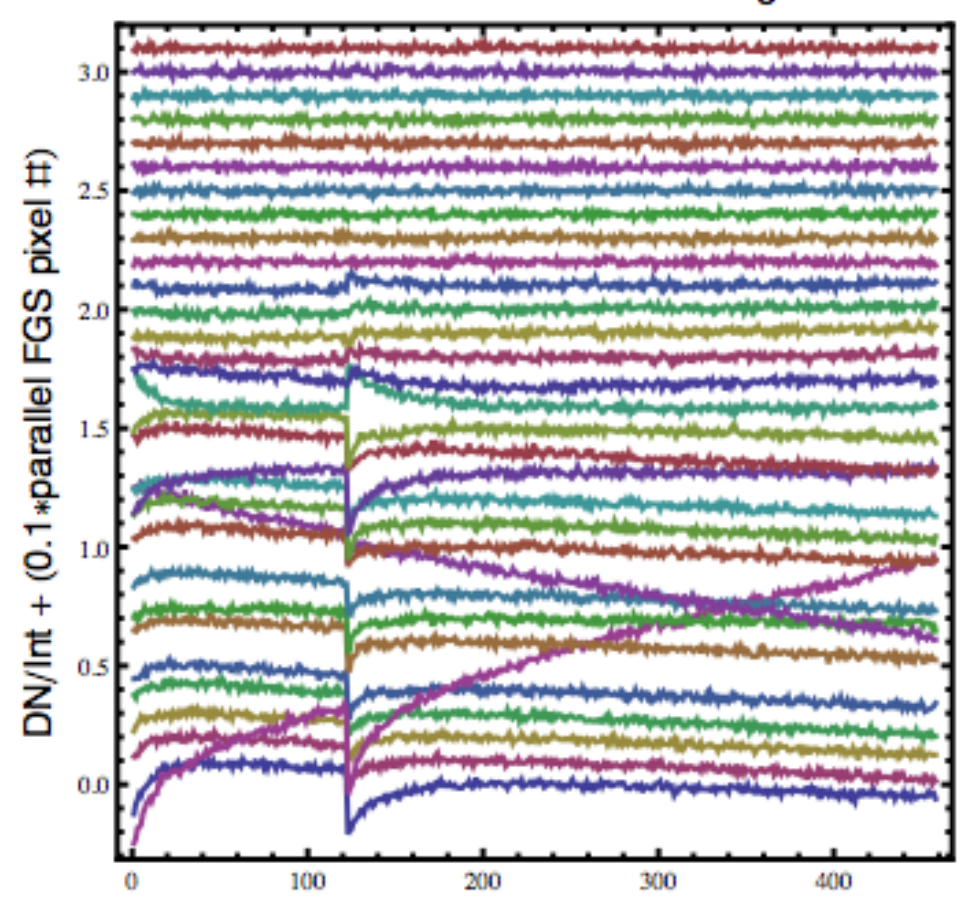

Mean of All DRV and ACQ BOARD Temperatures

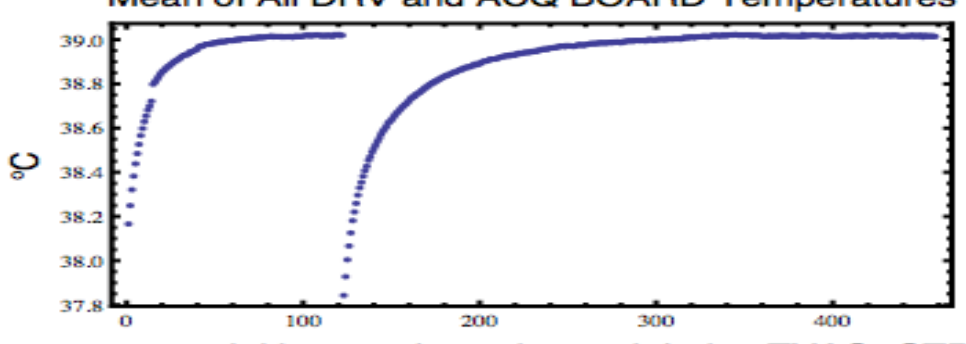

sequential long cadence interval during TVAC-CTB

SPIE:Astronomical Telescopes and Instrumentation 2010 


\section{High Frequency Signal Alias}

- High frequency $(\geq 1 \mathrm{GHz})$ amplifier oscillations are aliased into the CCD readout $(3 \mathrm{MHz})$

- Aliased signal changes frequency due to temperature dependence of primary signal

Row-by-Row FFT2 of CCD image difference

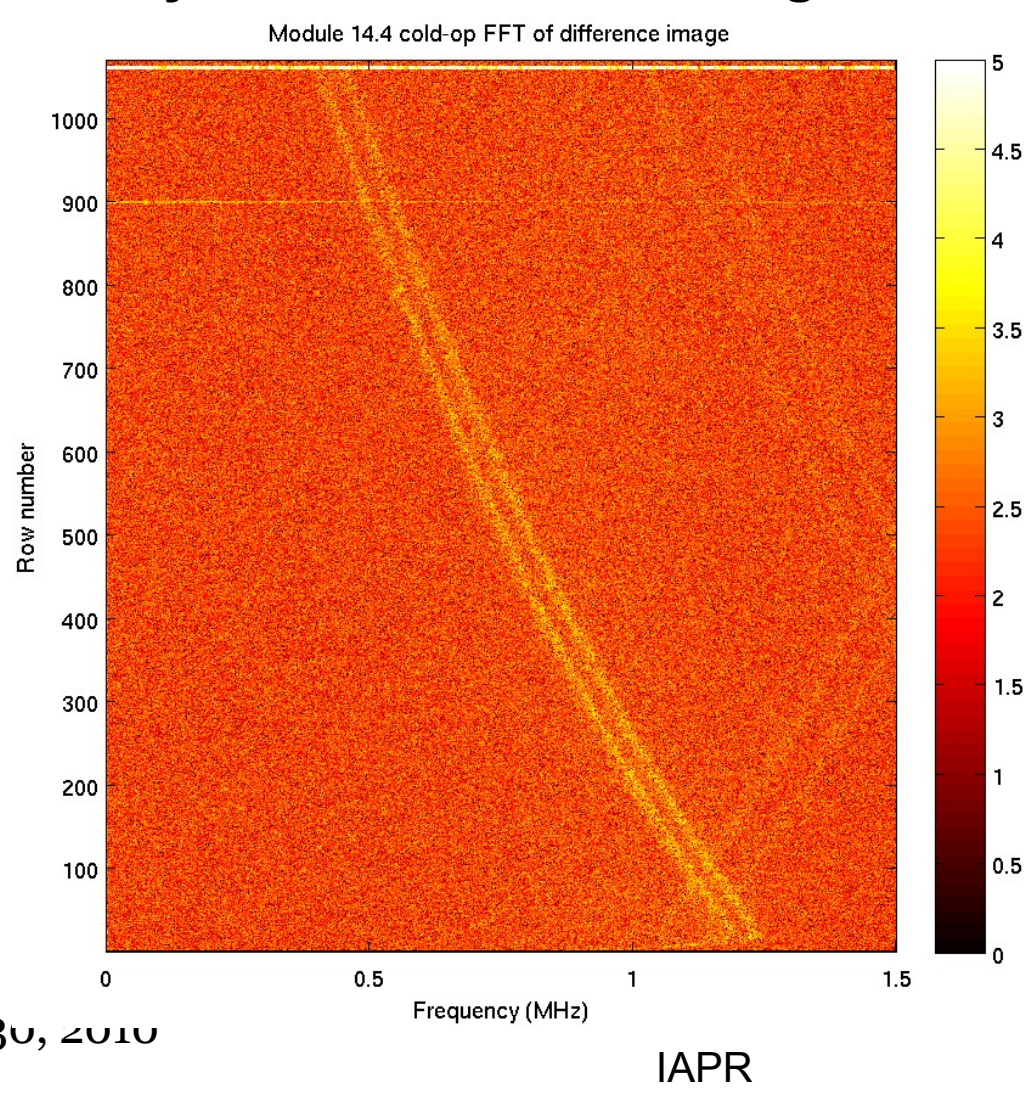

Time evolution of |FFT| of one row

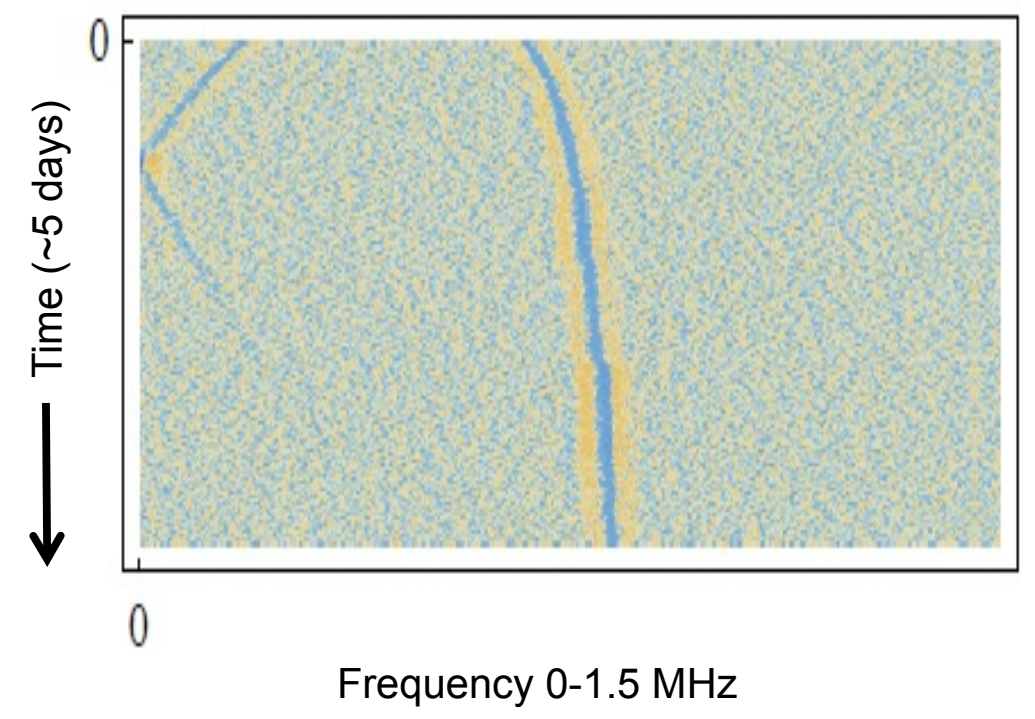




\section{High Frequency Signal Alias}

- Rolling Band Artifact (RBA)

- Rolling band is a manifestation of the high frequency oscillation signal aliased to $\mathrm{DC}$ (multiples of $3 \mathrm{MHz}$ read-out rate)

- RBAs move on the image as the oscillation frequency changes with temperature

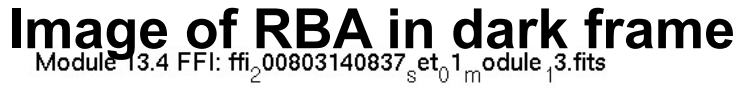

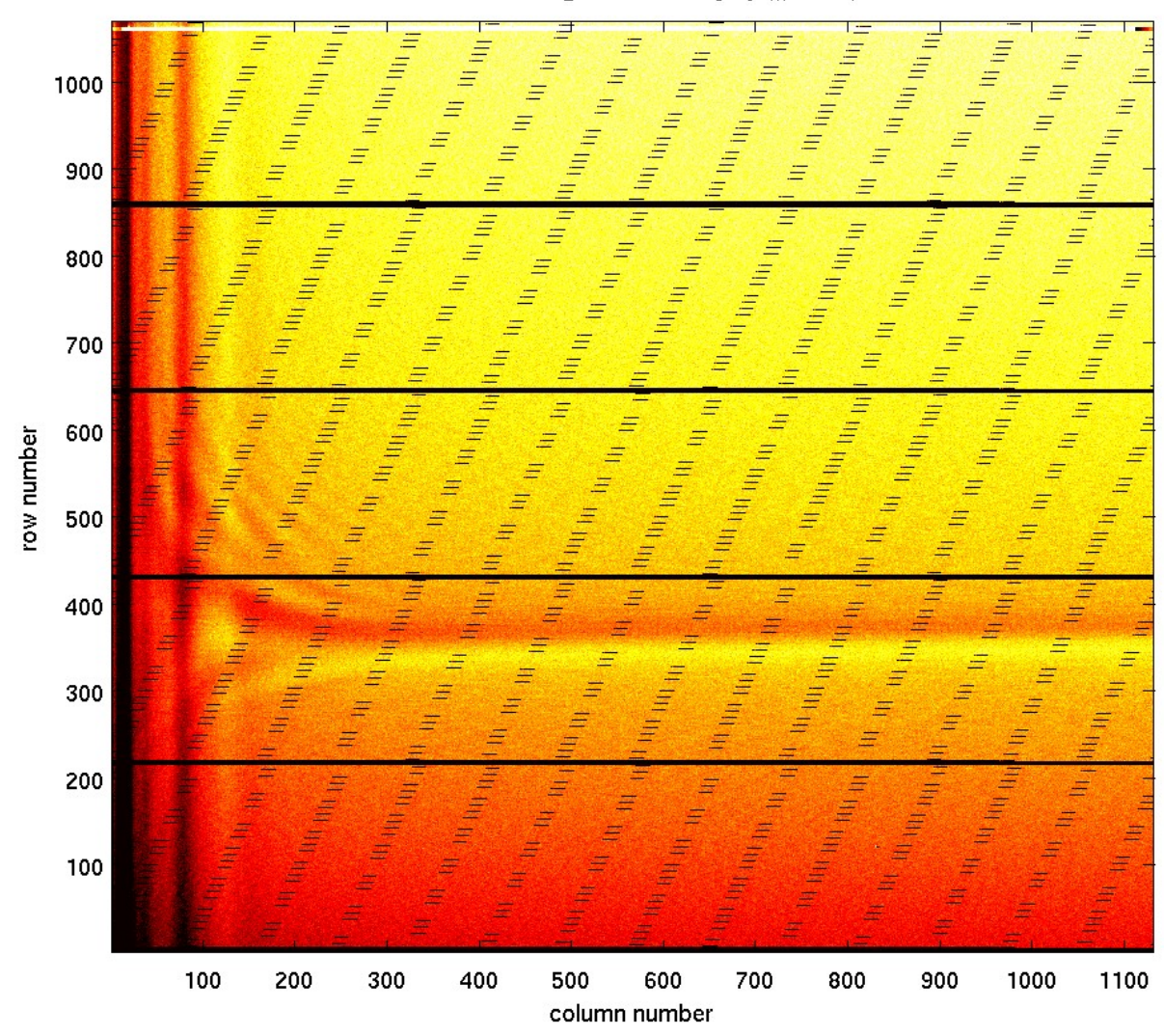

Jun 30,2010

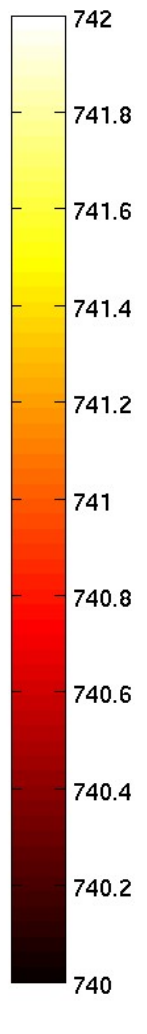

Time evolution of RBA measured in trailing black

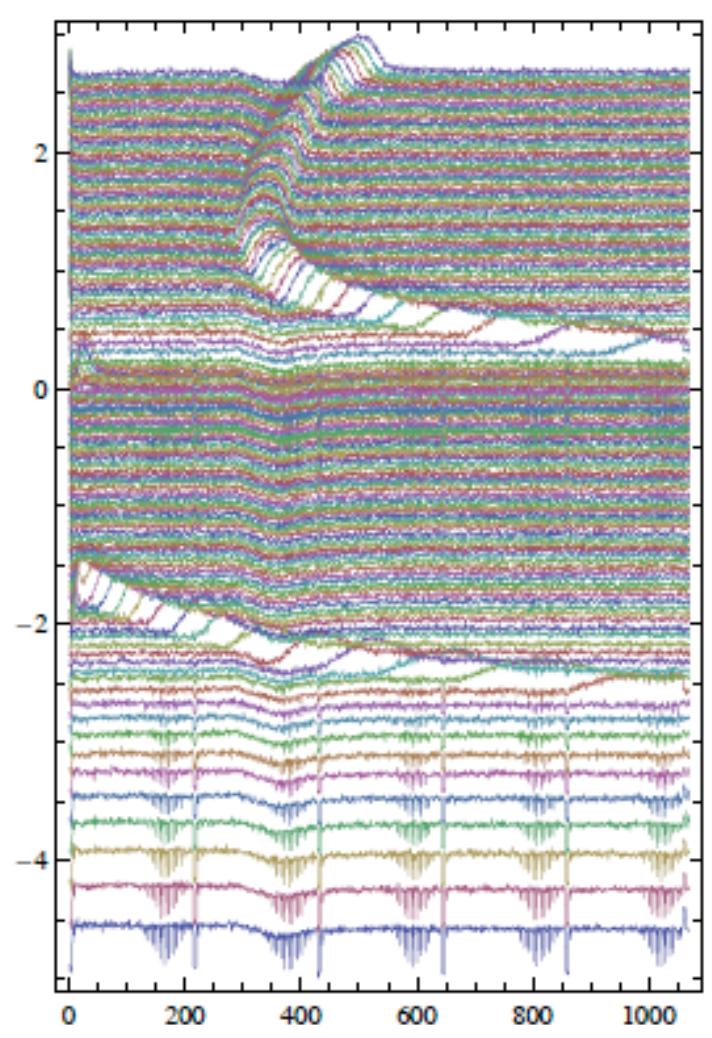

SPIE:Astronomical Telescopes and Instrumentation 2010 


\section{High Frequency Signal Alias}

Kepler

Moiré Pattern Drift (MPD): high frequency oscillation aliased into the CCD read-out. The pattern becomes more pronounced as the temperature stabilizes
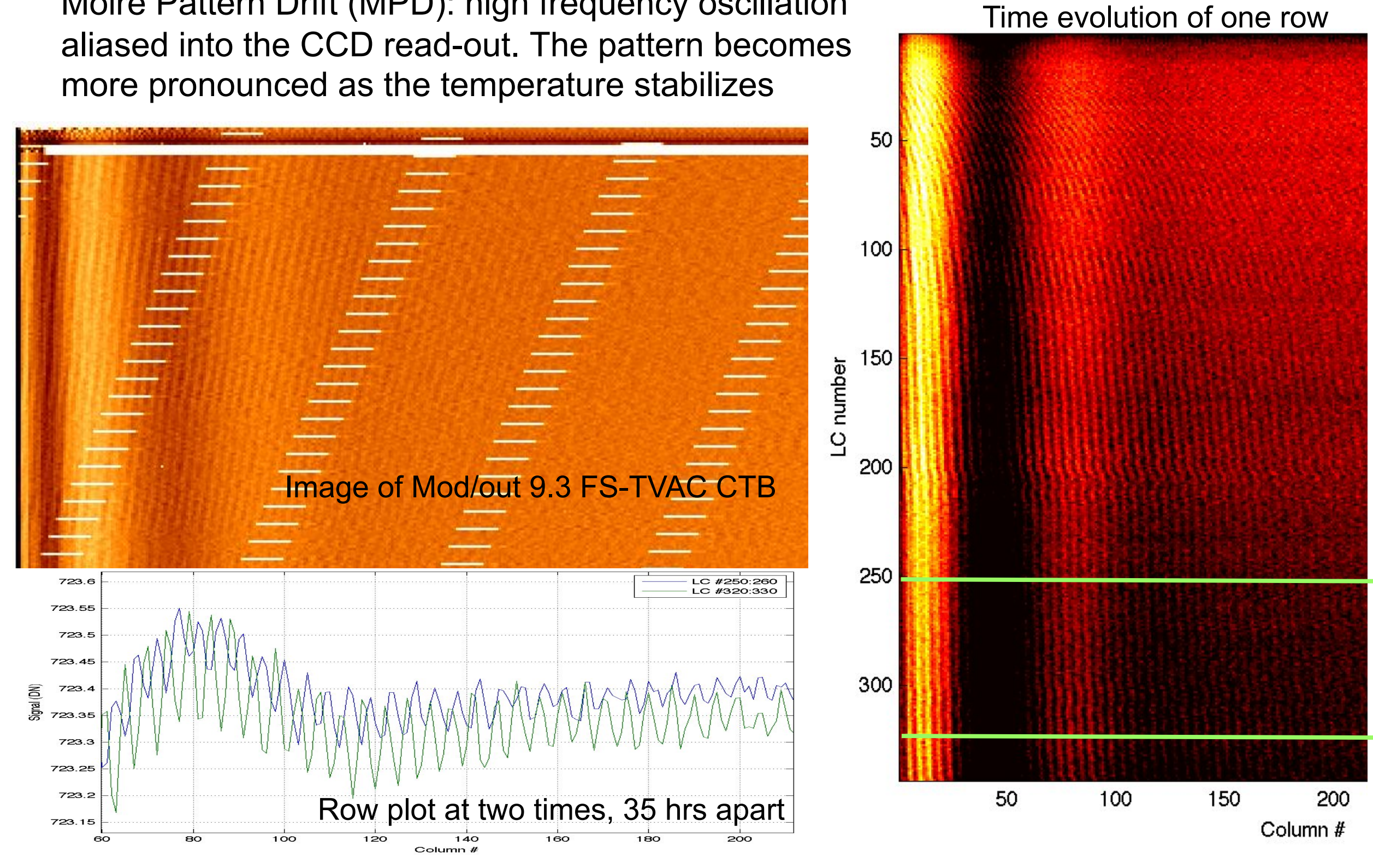


\section{Effects and Risks}

- Reasons for concern

- Kepler is searching for an 84 ppm signal from a source generating $190000 \mathrm{e}^{-} / \mathrm{sec}^{-}$ in an aperture of $\sim 17$ pixels.

- A time-varying, spatially-correlated instrument-induced bias of $<1 \mathrm{e}^{-} / \mathrm{sec} / \mathrm{pixel}$, could produce, prevent or complicate the detection of such a signal.

- If the time signature is locally correlated but becomes uncorrelated at larger image distances, it may become impossible to remove the bias.

- Instrument-induced effects meeting both of these criteria exist on some Kepler channels.

- Maximum acceptable bias variations

\begin{tabular}{|l|l|}
\hline signal from $12^{\text {th }}$ magnitude star in 6.5 hours & $4.47 \times 10^{9} \mathrm{e}^{-}$ \\
\hline earth-size planet transit decrement $84 \mathrm{ppm}$ & $376000 \mathrm{e}^{-}$ \\
\hline allocated noise level $20 \mathrm{ppm}$ & $89500 \mathrm{e}^{-}$ \\
\hline $\begin{array}{l}\text { per-pixel per-read bias change equivalent to } 20 \mathrm{ppm} \\
89500 \mathrm{e}^{-} / 16 \text { pixels / } 270 * 13 \mathrm{read}\end{array}$ & $1.6 \mathrm{e}^{-} / \mathrm{pixel} / \mathrm{read}$ \\
\hline $\begin{array}{l}\text { RE read / long cadence, } 13 \text { long cadence } / 6.5 \mathrm{hr} .) \\
\text { Chosen by rounding up }\end{array}$ & $\begin{array}{l}2 \mathrm{e}^{-} / \mathrm{pixel} / \mathrm{read} \\
0.02 \mathrm{DN} / \mathrm{pixel} / \mathrm{read} \\
(25 \mathrm{ppm})\end{array}$ \\
\hline
\end{tabular}

- This is the amplitude where artifacts begin to become significant

- $\quad$ need to then investigate the time signature 


\section{Objectives}

- Goal: Appropriately account for artifacts in the Kepler data

- Know when, where and at what sensitivity level artifacts are important and unimportant.

- Correct data where effects can be observed directly.

- Enable complete and efficient neutralization of artifacts assuming suitable additional steps in later stages of processing.

- Objective 1: Remove pixel-dependent FGS crosstalk variations and lowspatial frequency variations.

- Ensures knowledge of sensitivity level where effects become important.

- Removes a known source of temperature dependent variation in pixel time series.

- Enhances confidence in accuracy of pixel time series over the long term and near $\mathrm{S} / \mathrm{C}$ thermal transients.

- Improves robustness against unpredictable processing results - may help to stabilize effectiveness of existing and future pipeline modules.

- e.g., potentially enhanced stability for robust fit parameters for black and background

- Objective 2: Detect, characterize, and flag rolling bands and moiré patterns.

- Also ensures knowledge of sensitivity level where effects become important.

- Enables neutralization using statistical weights or sample exclusion zones based on observed characteristics.

- Secondary Objectives: Provide means to monitor uncorrected artifacts and evaluate scene-dependence 


\section{Available Data}

- Collateral Data

- Trailing Black (Virtual Columns)

- Summed across 14 columns (1119-1132)

- Masked and Virtual Smear (Rows)

- Summed over 12 rows each (7-18, 1047-1058)

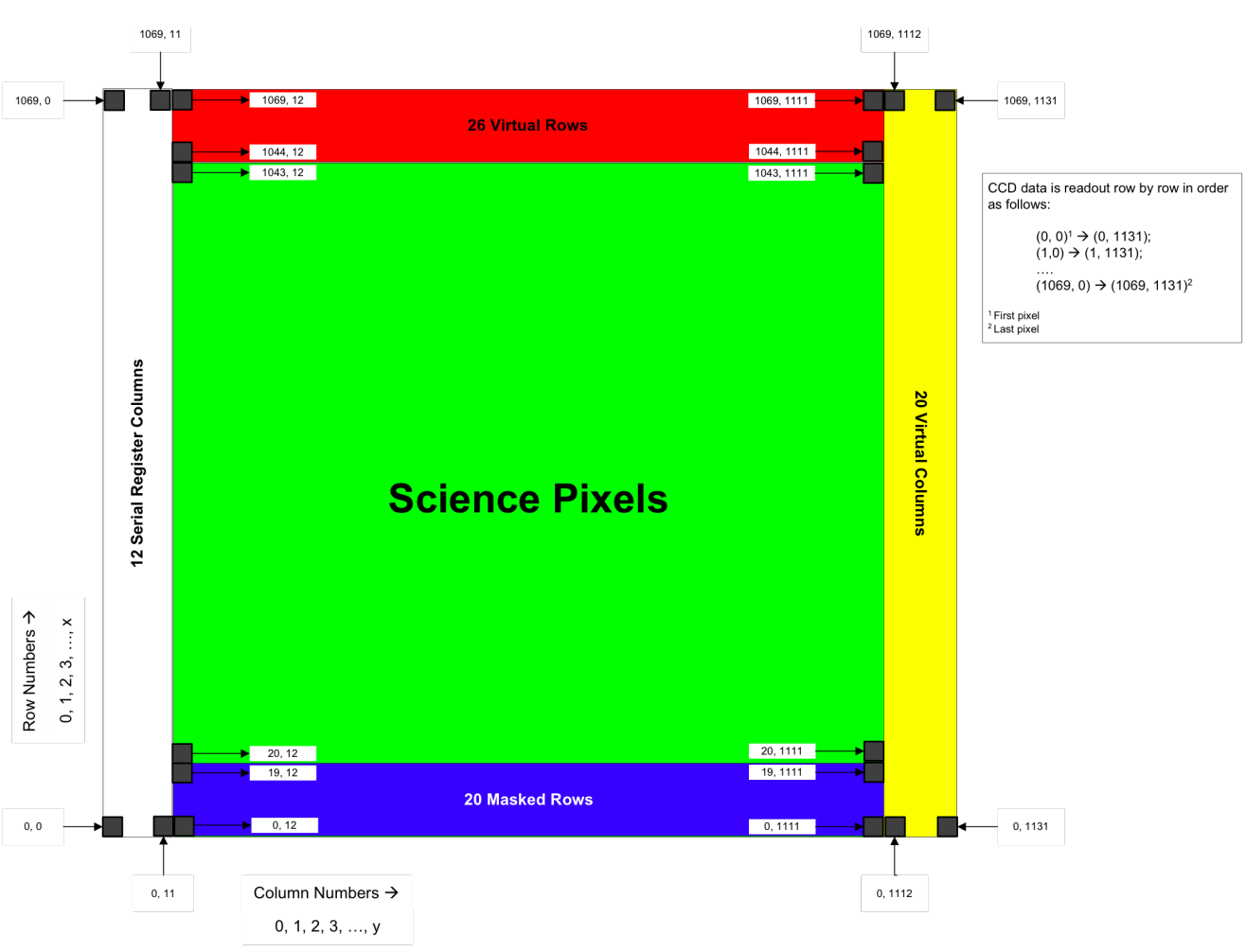

- Artifact Removal Pixels (ARPs)

- Cover time-varying FGS crosstalk

- Also permit undershoot measurement following charge injection rows 1060-1063)
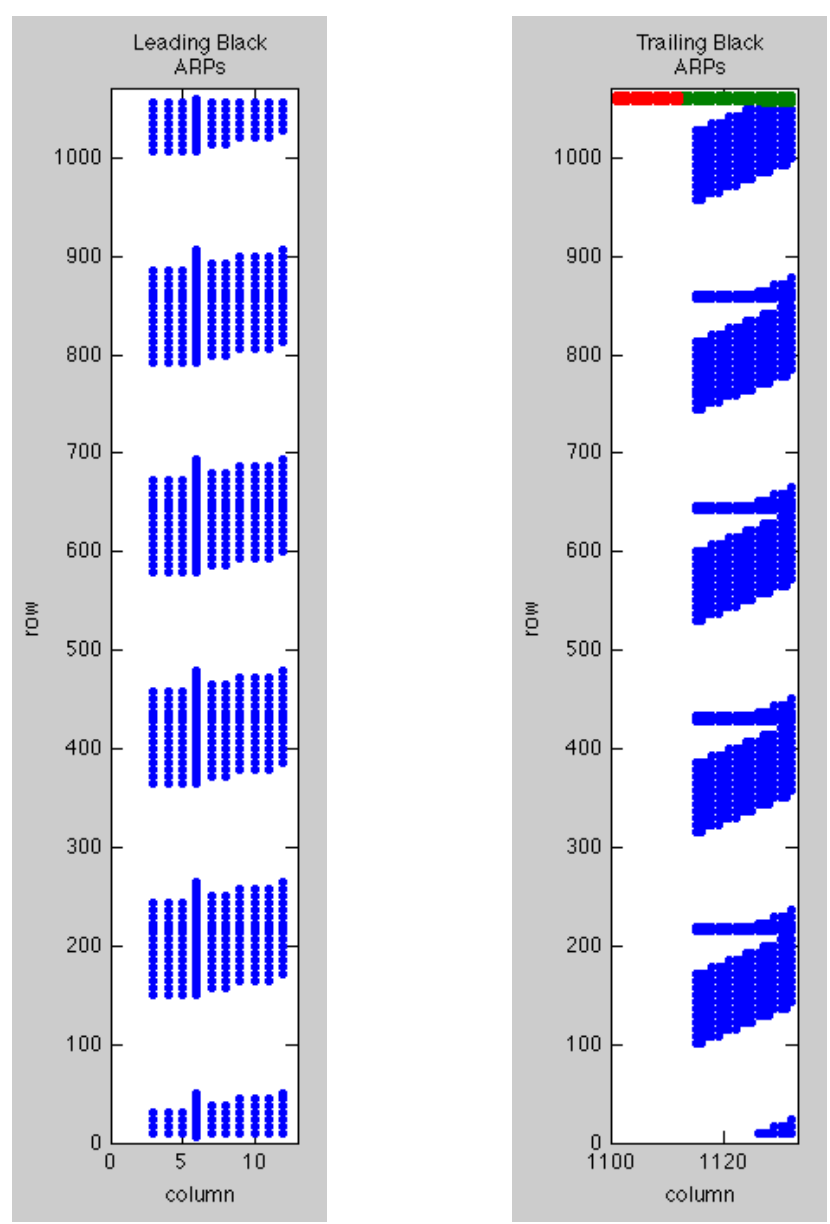


\section{Prototype Architecture Overview}

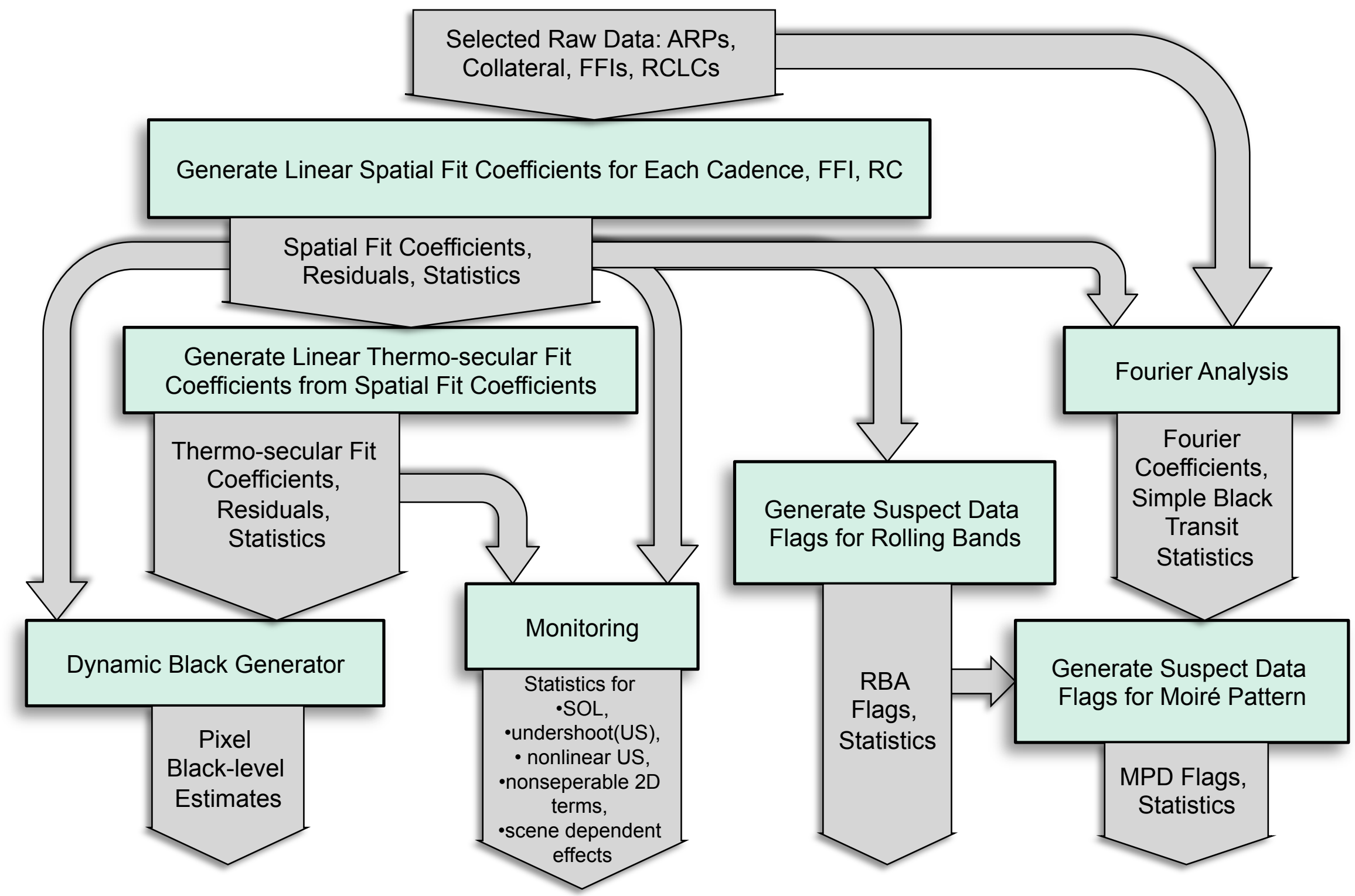




\section{Formal Model Definition}

- Let $X_{R C}$ represent the raw black level value in $\mathrm{DN}$ at row $\mathrm{R}$ and column $\mathrm{C}$, and let $Z$ represent the zero offset introduced to prevent negative values

$X_{R C}-Z=X_{0}+f_{R C}+g_{C}+W_{R C}^{F}+W_{R C}^{P}+U_{R C}$

where,

$X_{0}$ is a constant

$f_{R C}$ is the row dependent part, (C only distinguishes leading and trailing black)

$g_{C}$ is the column dependent part

$W_{R C}^{F}$ is the FGS frame crosstalk dependent part

$W_{R C}^{P}$ is the FGS parallel crosstalk dependent part

$U_{R C}$ is the undershoot dependent part 


\section{Example}

- Serial pixel row dependence:
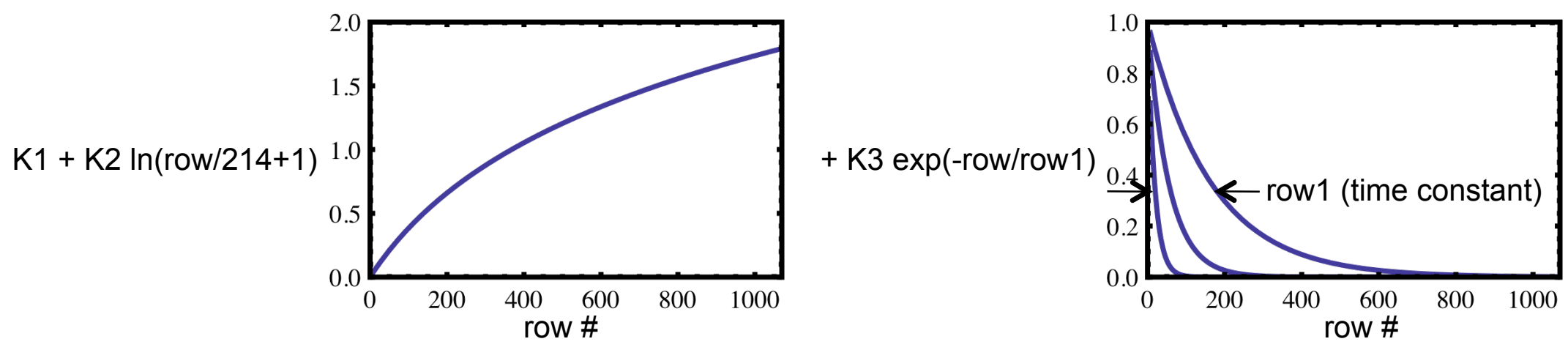

- row 1 parameter is determined by a nonlinear fit to an FGS corrected response vector derived from the mean of all cadences.
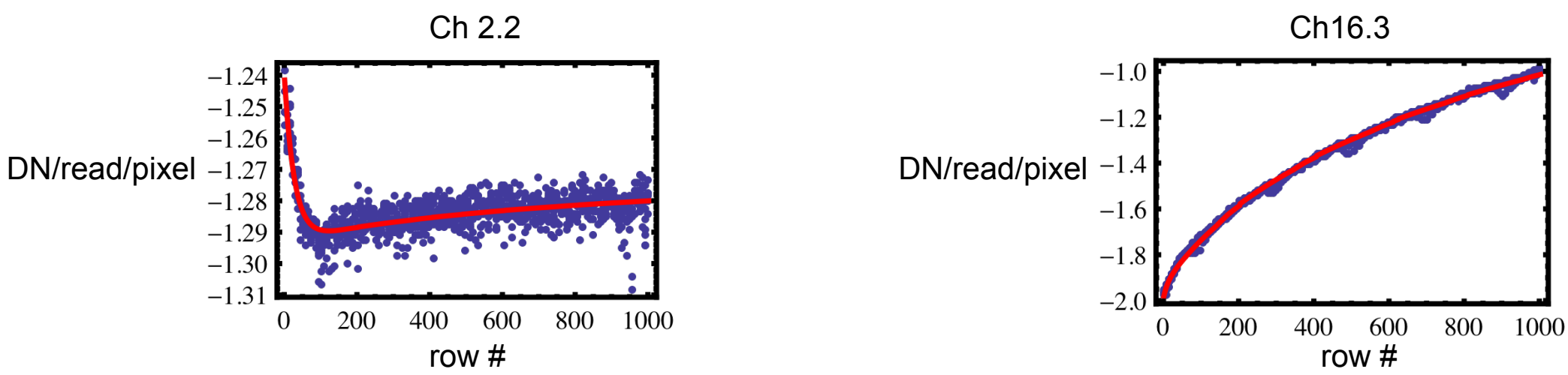

- A collateral data point contains 14 serial pixel values (trailing black columns) so corresponding serial row model elements are scaled by a factor of $14 \mathrm{x}$.

- Collateral portion is de-weighted by sqrt(14) to equalize per-point noise. 


\section{Examples: Qo-Q1 Ch. 2.1, 20.2}

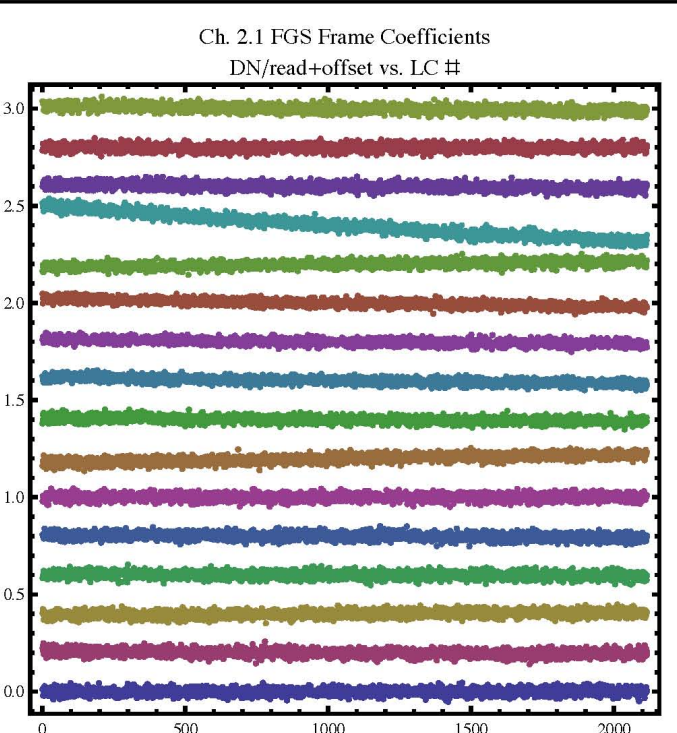

Ch. 2.1 FGS Parallel Coefficients DN/read+offset vs. LC

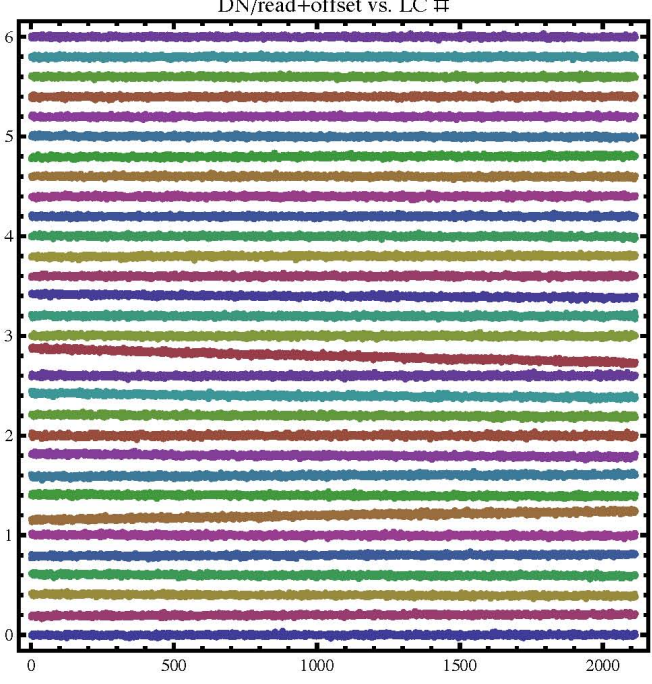

Jun 30, 2010

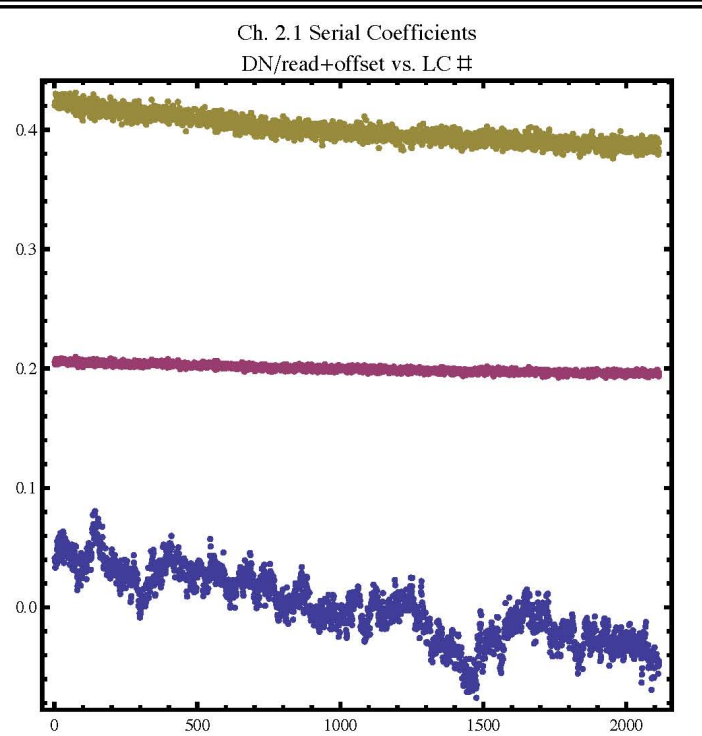

Ch. 20.2 Serial Coefficients

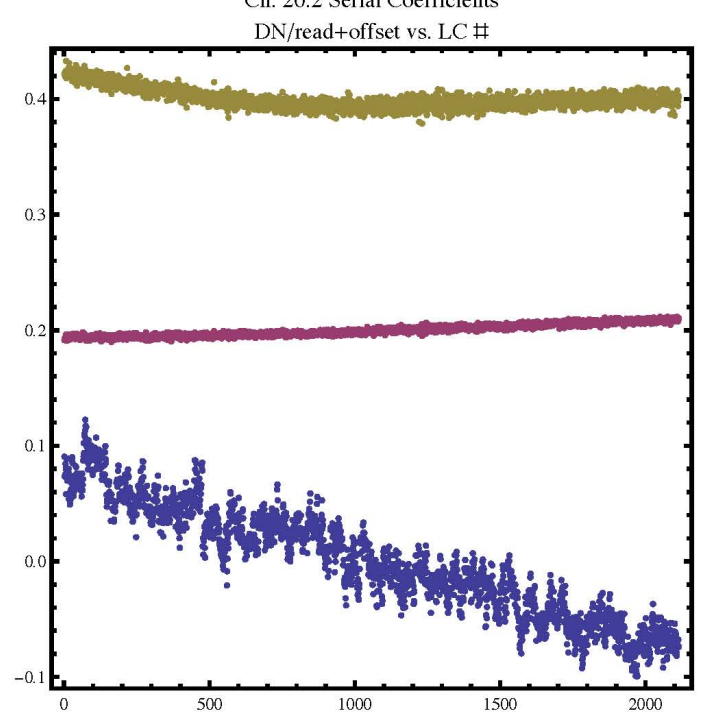

12

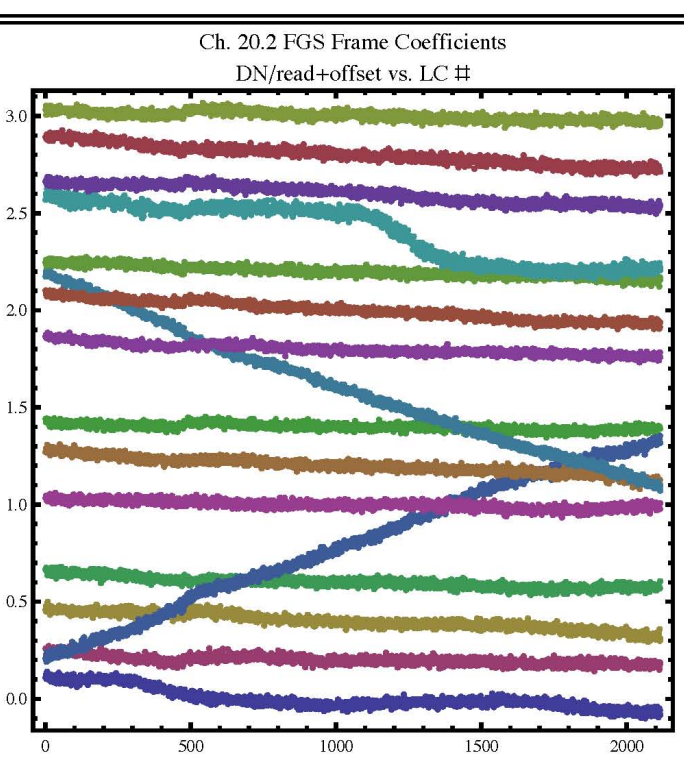

Ch. 20.2 FGS Parallel Coefficients

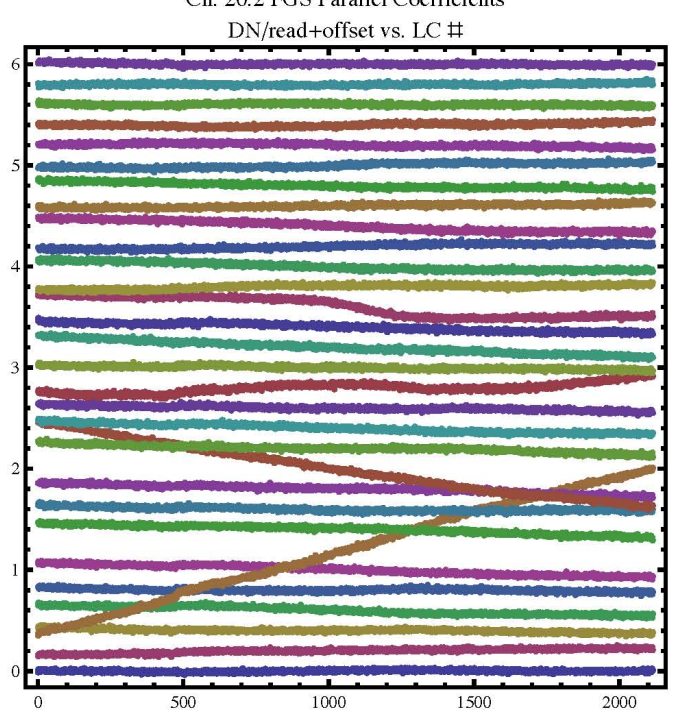

SPIE:Astronomical Telescopes and Instrumentation 2010 


\subsection{Trailing Black}

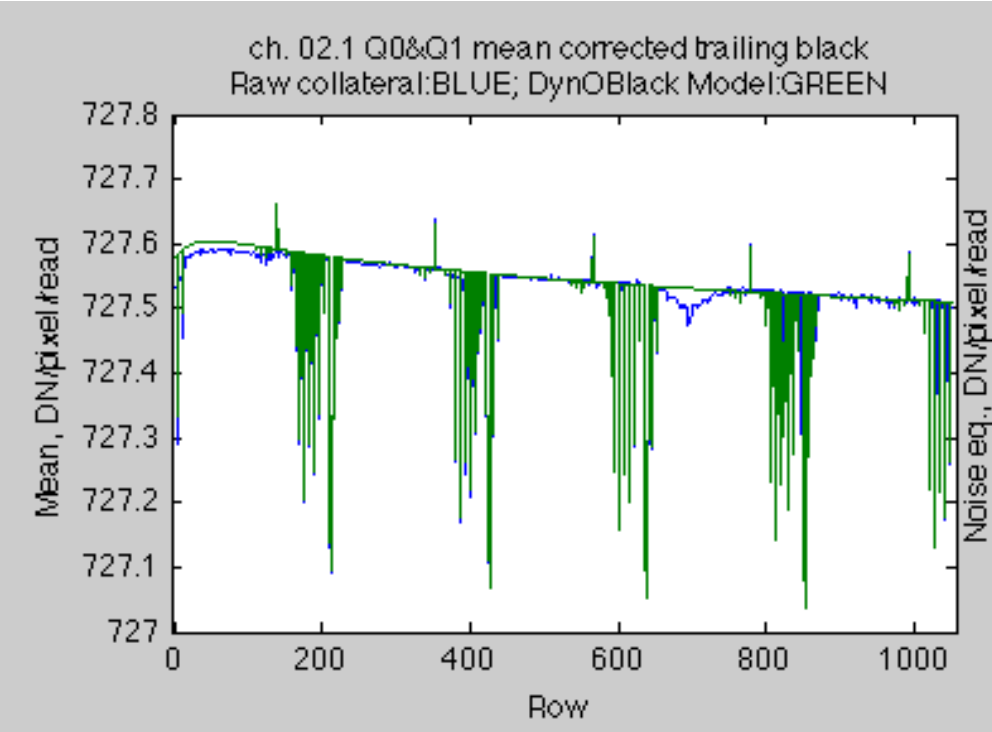

Noise level using $68 \%$-width/2 (also *1/sqrt $270 \star 14$ )) Dynoblack Model: BLUE, Raw: RED, Raw-Dynoblack: BLACK
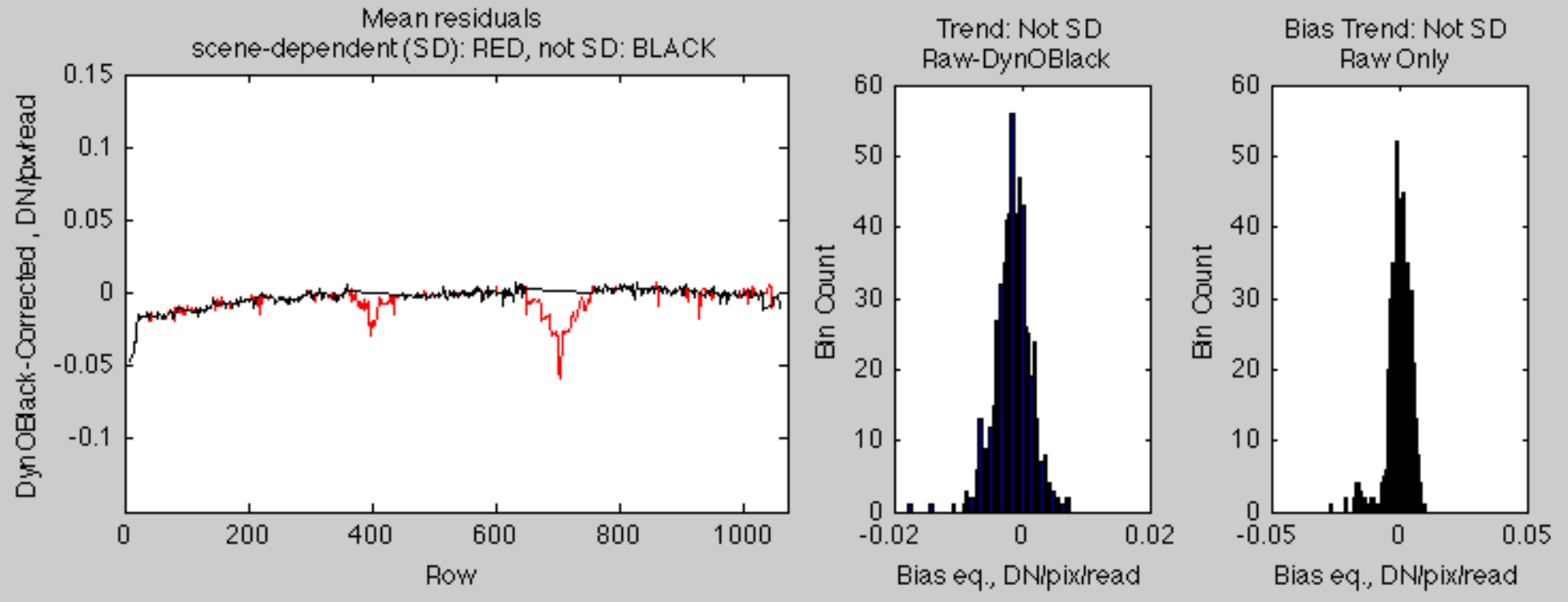


\section{Channel Summary, CDPP + Q1M1}

- Test channel result summary: blue regions are not flagged orange/gold are preliminary flags white are expanded regions of final flags

\begin{tabular}{|c|c|c|}
\hline Channel & flag count & \% flagged \\
\hline 2.1 & 0 & 0 \\
\hline 6.2 & 0 & 0 \\
\hline 9.2 & 114 & 39.9 \\
\hline 12.1 & 0 & 0 \\
\hline 17.2 & 436 & 18.3 \\
\hline 20.2 & 0 & 0 \\
\hline
\end{tabular}

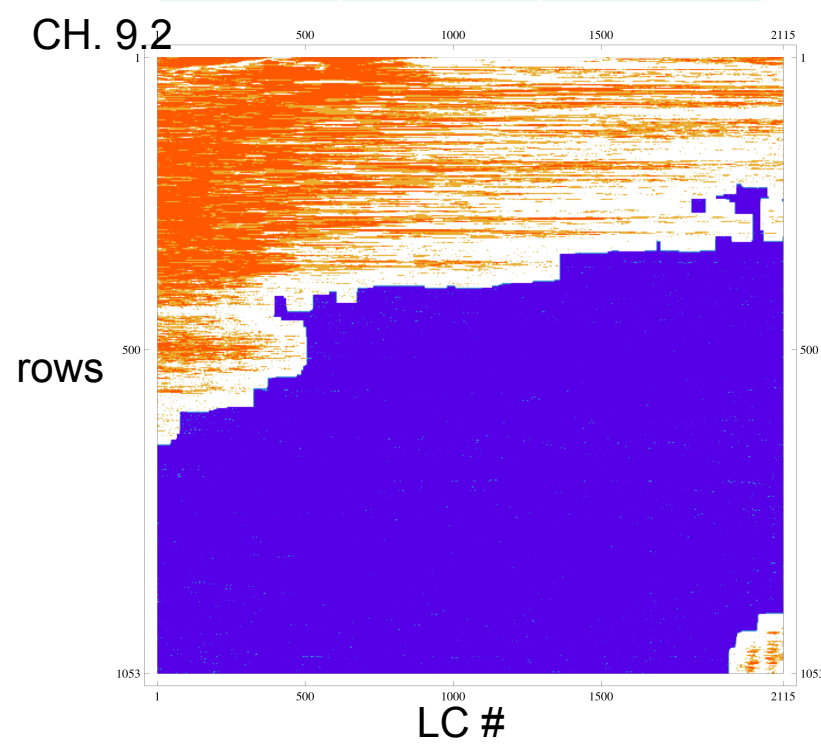

row \# - 6

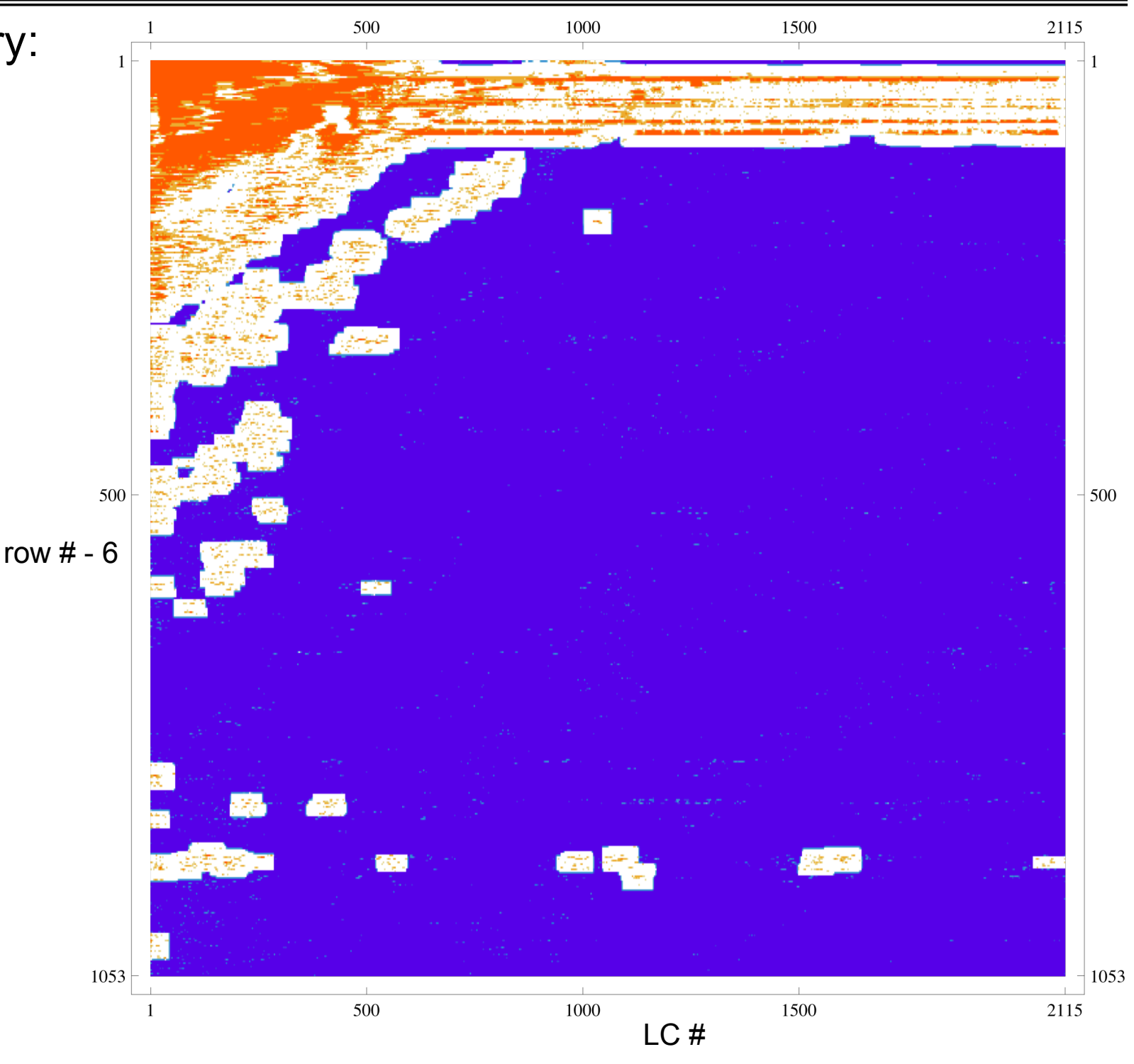




\section{Assumptions and Strategy}

- Facts and Assumptions for Moiré pattern flagging

- The discriminator for Moiré Pattern is a continuously changing frequency with temperature and/or time.

- Spatial bias modulation vs. column translates into bias variations over time in varied and complex ways.

- affected by scene elements, bad columns/pixels, step change at start and end of lines

- also affected by thermal transients, and long term rate of temperature change

- Moiré Pattern not directly measurable cadence-by-cadence vs. row and column

- The presence of Moiré Pattern in specific columns of smear collateral may propagate into all rows in CAL.

- Strategy

- Rely on spatial variation for Moiré pattern flagging as an indicator of local timevariability.

- Combine FFI spatial information with Collateral time-dependent information to get cadence-by-cadence vs. row and column

- Generally err toward the worst case.

- Collect extra information with goal to help improve understanding over time. 


\section{Fourier Analysis - FFI}

\section{- $\quad$ Ch. 9.2 Q2-09 - 2 FFIs}

- $\quad$ robust fit residuals, (FFI- mean $\mathrm{FFI}$ )

- row-by-row FFT

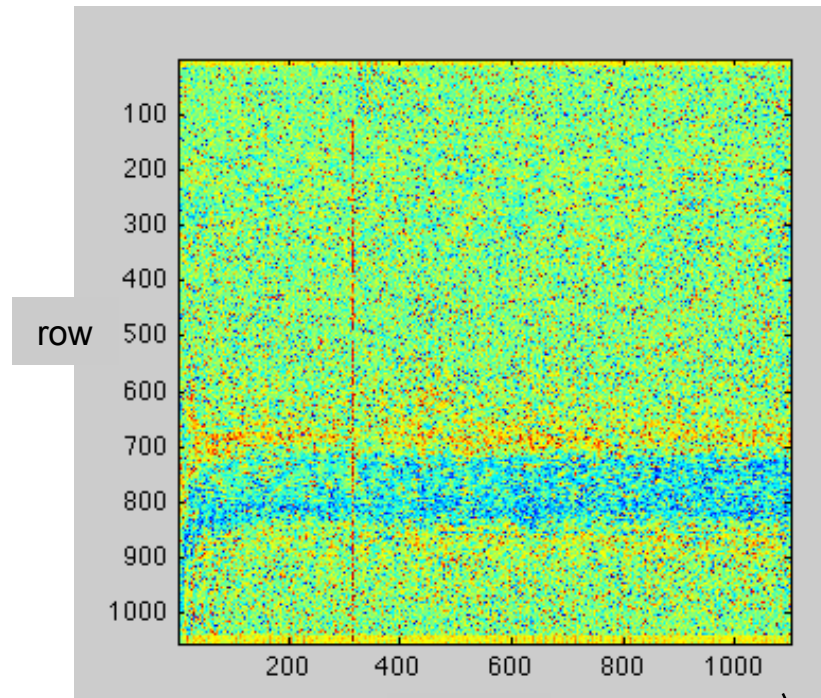

column

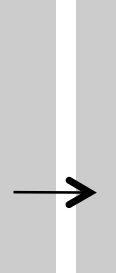

row
FFT bins

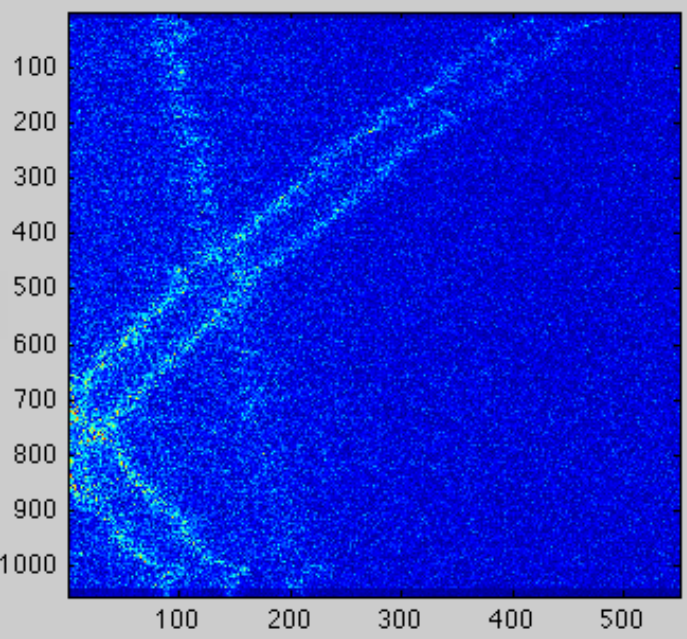

- local 2D averaged

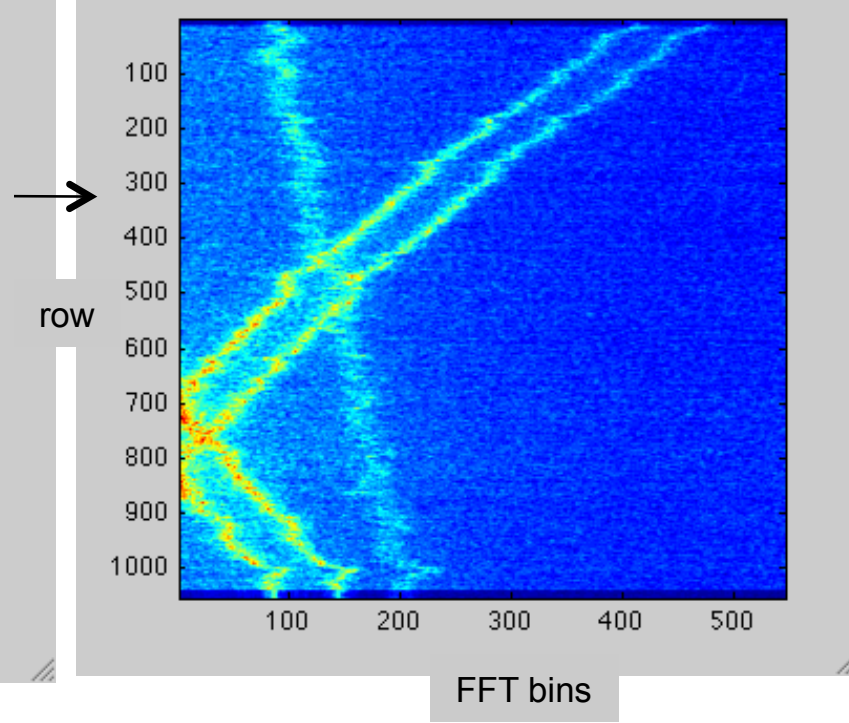

noise level is above intrinsic noise due to limitations of outlier rejection all FFT bins range from 0 to $1.5 \mathrm{MHz}$ or 0 to 0.5 pix $^{-1}$

- $\quad$ windowed row-by-row FFT
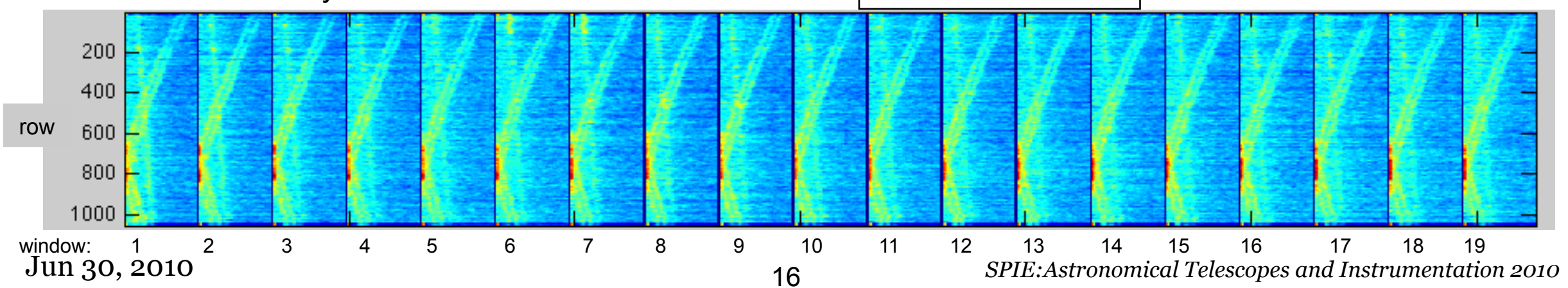

16 


\section{Example: channel 9.2, CDPP + Q1M1}

- FFI traceable peaks:
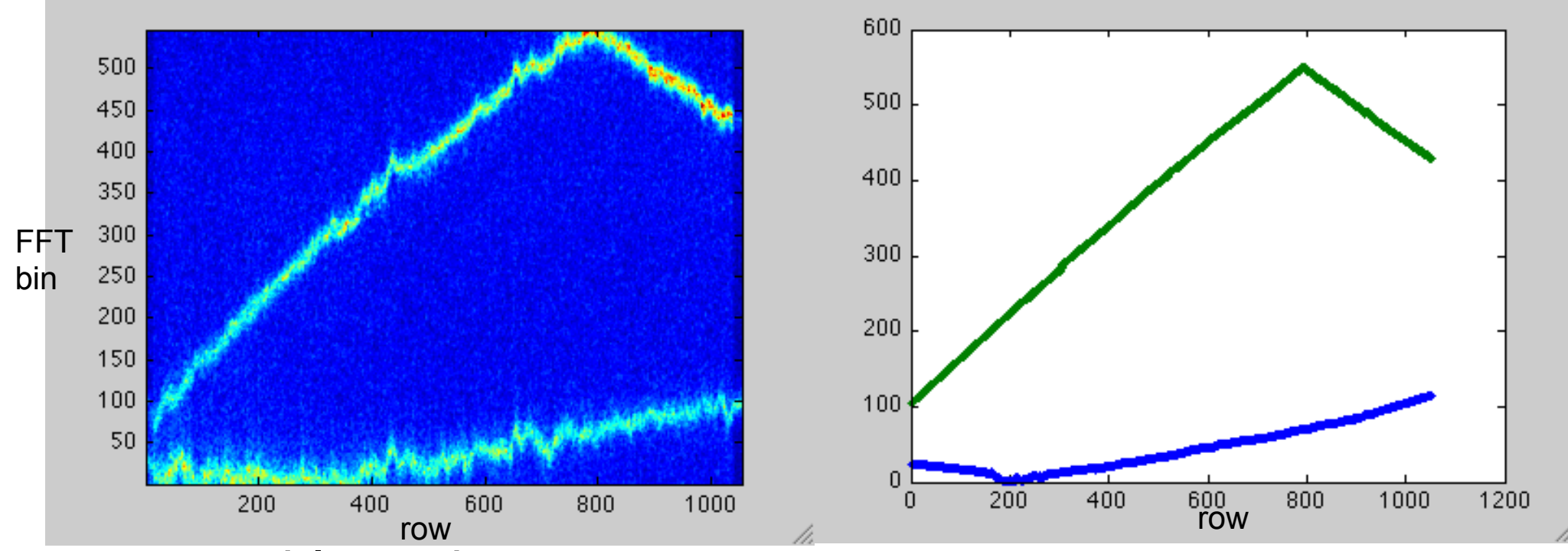

- Smear traceable peaks:

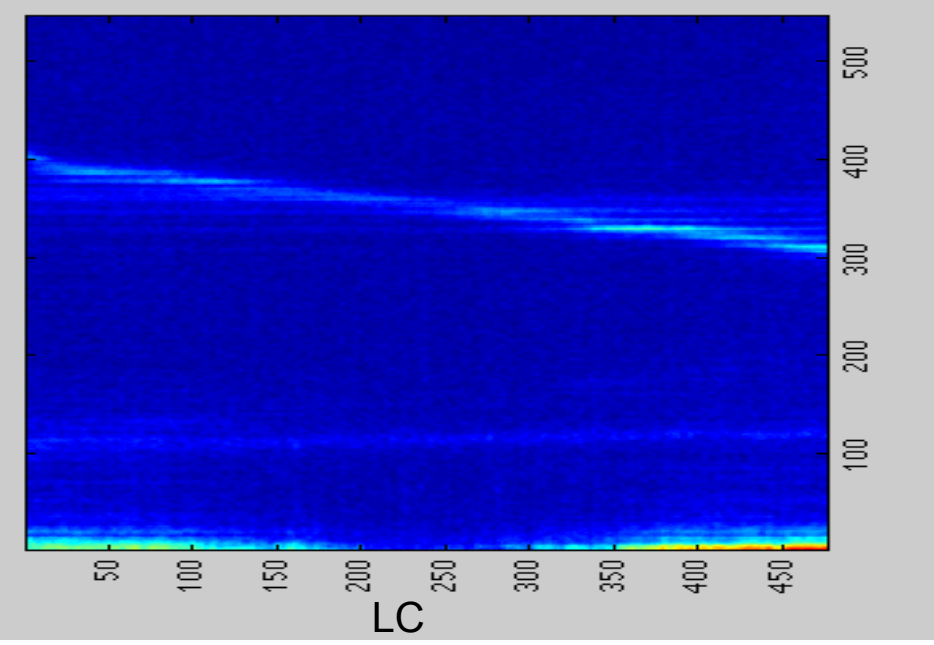

17

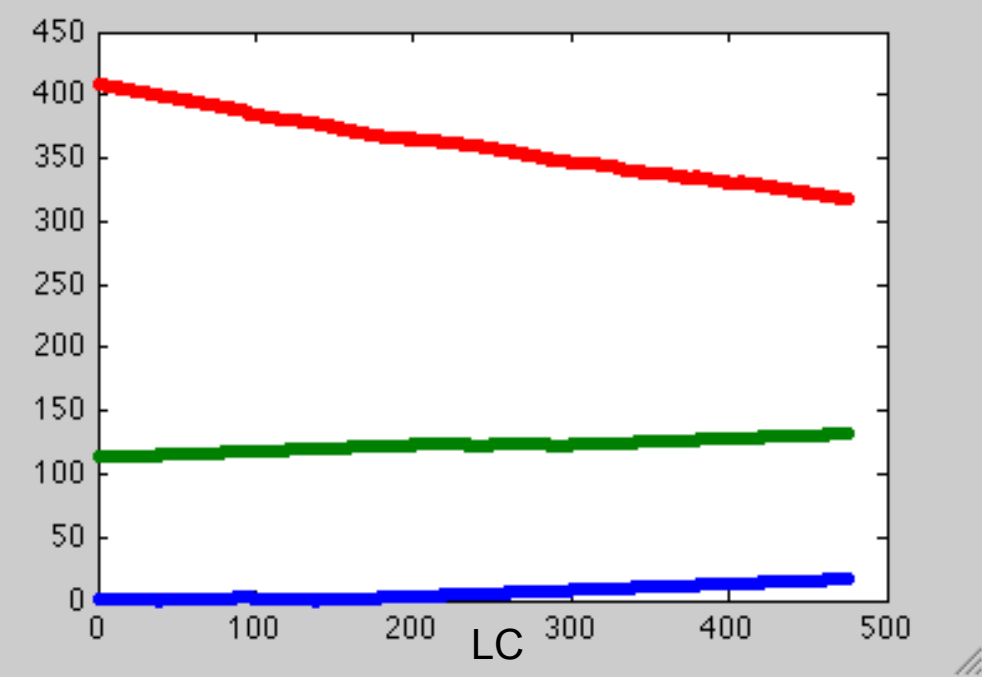

SPIE:Astronomical Telescopes and Instrumentation 2010 


\section{Effectiveness summary}

- Results based on Q1 results from all channels are consistent with expectations

- FGS variation effect on targets

- Trends: $3.7 \%$ of targets above $0.02 \mathrm{DN} /$ Read in 35 days with $1^{\circ} \mathrm{C}$ temperature change;

- $17.4 \%$ of targets' optimal apertures include modeled FGS clock intervals

- Noise: negligible effect

- Rolling Bands

- 9 RBA channels; $3.0 \%$ of FOV at a given time during first 10 days of Q1

- Moire Pattern

- MPD 19 channels; $12.7 \%$ of FOV at a given time during first 10 days of Q1

Fraction of targets with $<.02$ DN/read FGS correction

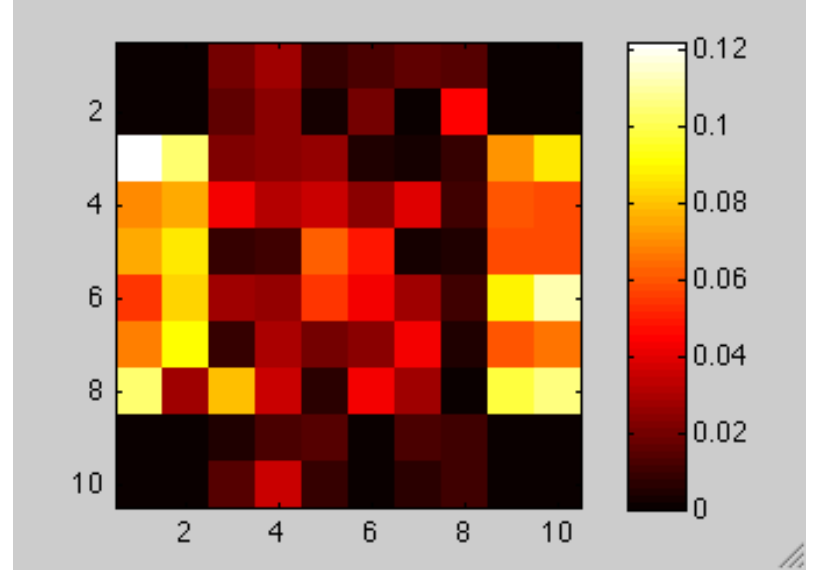

Flagged RBA channels

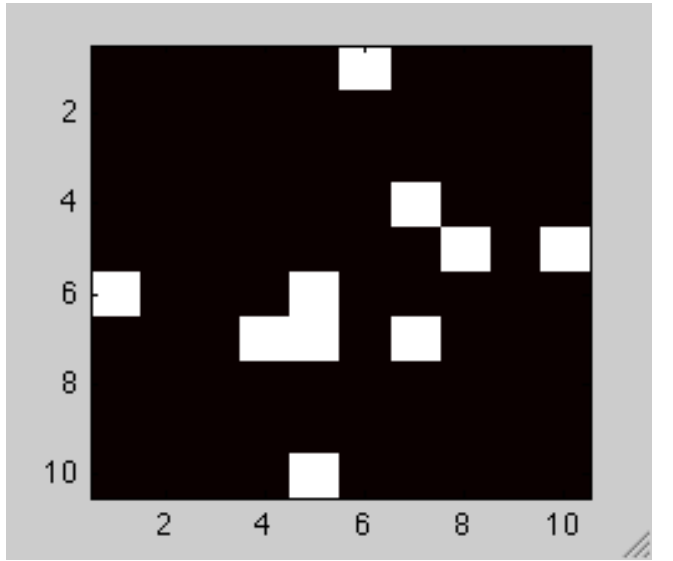

Flagged MPD channels

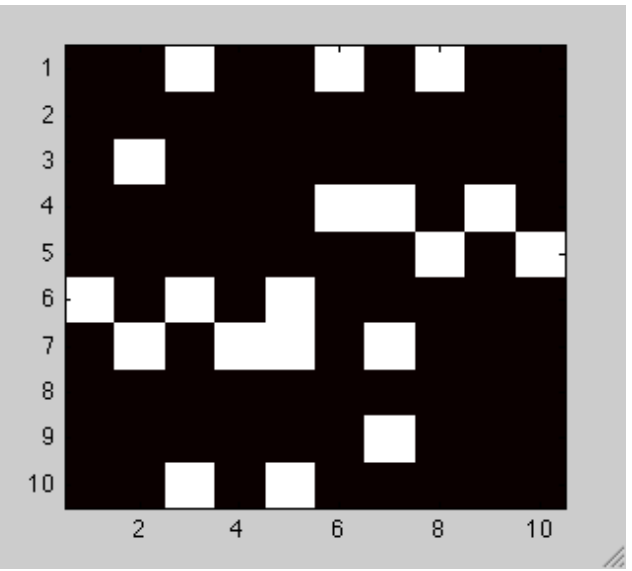

